
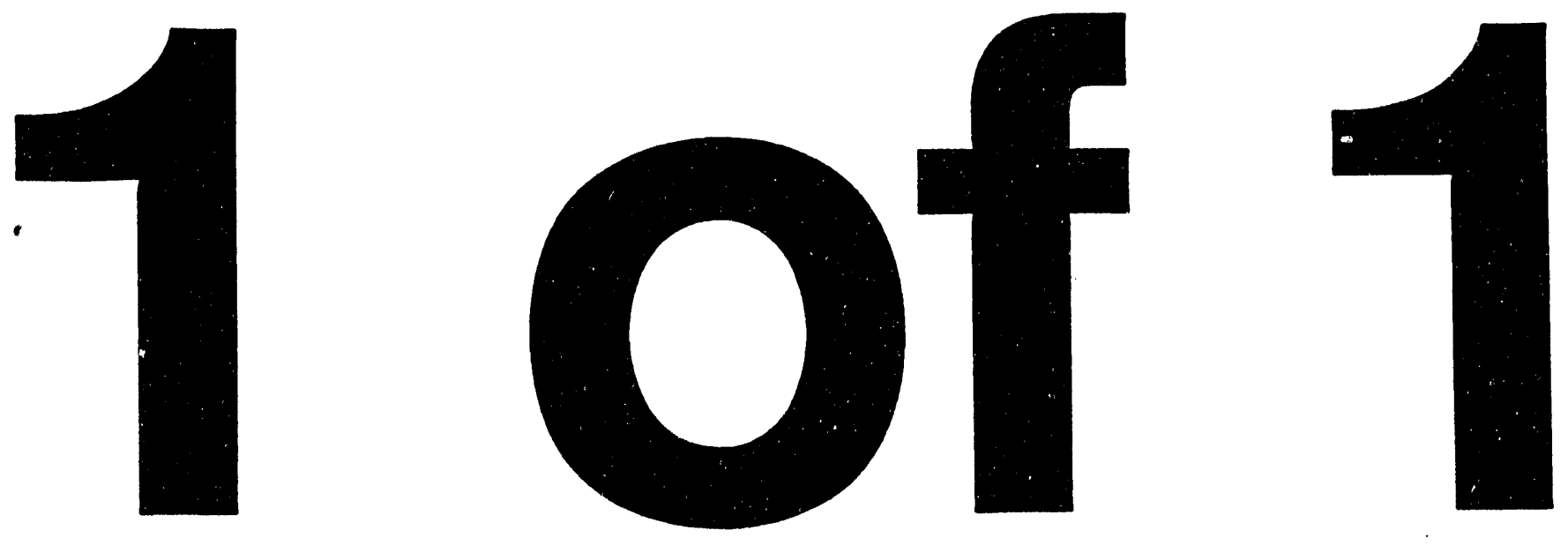
LBL-34243

UC - 1600

\title{
TECHNOLOGY DATA CHARACTERIZING LIGH TING IN COMMERCIAL BUILDINGS: APPLICATION TO END-USE FORECASTING WITH COMMEND 4.0
}

A. Osman Sezgen, Y. Joe Huang, Barbara A. Atkinson, Joe H. Eto and Jonathan G. Koomey

\author{
Energy Analysis Program \\ Energy and Environment Division \\ Lawrence Berkeley Laboratory \\ Iniversity of California \\ Berkeley, CA 94720
}

May 1994

This work was supported by the Assistant Secretary for Energy Efficiency and Renewable Energy, Office of Planning and Analysis and Office of Building Technologies, and the Office of Environmental Analysis, Office of Policy, Planning, and Analysis of the U.S. Department of Energy under Contract No. DE-AC03-76SF00098.

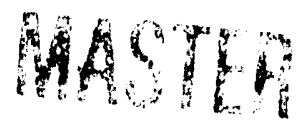




\title{
Technology Data Characterizing Lighting in Commercial Buildings: Application to End-Use Forecasting with COMMEND 4.0
}

\begin{abstract}
End-use forecasting models typically utilize technology tradeoff curves to represent technology options available to consumers. A tradeoff curve, in general terms, is a functional form which relates efficiency to capital cost. Each end-use is modeled by a single tradeoff curve. This type of representation is satisfactory in the analysis of many policy options. On the other hand, for policies addressing individual technology options or groups of technology options, because individual technology options are accessible to the analyst, representation in such reduced form is not satisfactory.

To address this and other analysis needs, the Electric Power Research Institute (EPRD) has enhanced its Commercial End-Use Planning System (COMMEND) to allow modeling of specific lighting and space conditioning (HVAC) technology options. The EPRI contractor for this effort, Regional Economic Research, Inc. (RER), worked with Lawrence Berkeley Laboratory (LBL) in the development and testing of the technology modules contained in COMMEND 4.0. LBL is also providing assistance in the development and refinement of technology data for the model.

This report characterizes the present commercial floorstock in terms of lighting technologies and develops cost-efficiency data for these lighting technologies. The report also characterizes the present lighting utilization patterns and lighting level requirements. Much of the data presented in this report were developed for the Analysis of Federal Policy Options for Improving U.S. Lighting Energy Efficiency, a study performed by LBL for the U.S. Department of Energy. This report organizes the data from the above-mentioned study in a form usable by a forecasting analyst.

This report also characterizes the interactions between the lighting and space conditioning end uses in commercial buildings in the U.S. In general, lighting energy reductions increase the heating and decrease the cooling requirements. The net change in a building's energy requirements, however, depends on the building characteristics, operating conditions, and the climate. Lighting/HVAC interactions data were generated through computer simulations using the DOE-2 building energy analysis program. Ten building types of two vintages and ten climate, were used to represent the U.S. commercial building stock for this purpose.
\end{abstract}




\section{Acknowledgments}

This report on commercial sector lighting is the first in a series summarizing technology data for various commercial end-uses in the United States. A companion report describes technology data for HVAC end-uses in office buildings. Reports due later in 1994 will characterize HVAC, water heating, office equipment, and refrigeration in all building types.

We would like to thank the analysts who reviewed this report, including Mohammad Adra of the Energy Information Administration in the U.S. Department of Energy, Ingrid Rohmund of Regional Economic Research, Inc., and James McMahon and Ellen Franconi of Lawrence Berkeley Laboratory.

The work was funded by David Patton of the Office of Planning and Assessment, Dick Jones of the Office of Building Technologies, and Ted Williams and John Conti of the Office of Policy, Planning, and Analysis in the U.S. Department of Energy. We are grateful for their support and insights.

This work was supported by the Assistant Secretary for Energy Efficiency and Renewable Energy, Office of Planning and Analysis and Office of Building Technologies, and the Office of Environmental Analysis, Office of Policy, Planning, and Analysis of the U.S. Department of Energy under Contract No. DE-AC03-76SF00098. 


\section{Table of Contents}

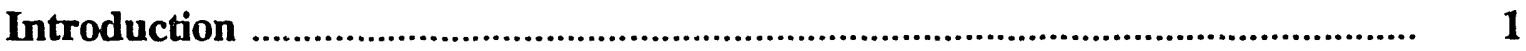

COMMEND Structure ……………………………….................................

Description and Development of Data ............................................................... 2

Equipment Definitions ........................................................................................... 2

Service and Electricity Demand .......................................................................

Service Demand-- Operating Hours and Lighting Levels ......................... 6

Room Factors .................................................................................... 7

EUI vs. Operating Hours ........................................................................... 7

Lighting Systems ........................................................................................... 9

Cost/Efficiency Data for Lighting Systems ................................................. 9

Saturation Data for Lighting Systems ....................................................... 24

Luminaire Data ....................................................................................... 33

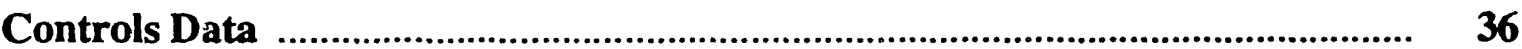

Lighting/HVAC Interactions ....................................................................... 40

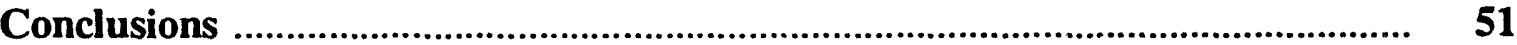

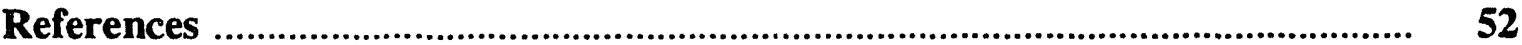




\section{List of Tables.}

Table 1. Systems with Fluorescent Fixture Types ....................................................

Table 2. Systems with Fixture Types other than Fluorescent ................................... 5

Table 3. Effective Lighting Hours (Annual) and Lighting Levels by Building

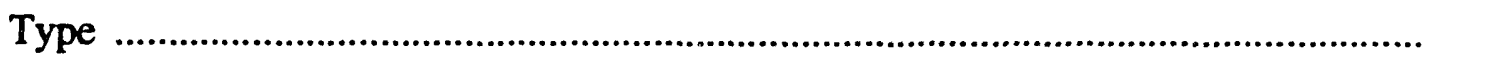

Table 4.a. Characteristics of 4-Foot Lamps with Energy-Efficient Magnetic

Ballast

Table 4.b. Characteristics of 4-Foot Fluorescent Lamps with Cathode-Cutout

Ballast

Table 4.c. Characteristics of 4-Foot Fluorescent Lamps with Electronic Ballast

Table 4.d. Characteristics of 8-Foot Fluorescent Lamps with Energy-Efficient

Magnetic Ballast

Table 4.e. Characteristic of 8-Foot Fluorescent High-Output Lamps with Energy-Efficient Magnetic Ballast

Table 4.f. Characteristics of 8-Foot Fluorescent Lamps with Electronic Ballast

Table 4.g. Characteristics of 8-Foot Fluorescent High-Output Lamps with Electronic Ballast

Table 5.a. Characteristics of General Service Incandescent Lamps ........................... 18

Table 5.b. Characteristics of Reflector Type Incandescent Lamps ........................... 19

Table 5.c. Characteristics of Compact Fluorescent Lamps ...................................... $\quad 20$

Table 6.a HID Lamps - 100W Mercury Vapor Lamps and Replacements ............... $\quad 21$

Table 6.b HID Lamps - 400W Mercury Vapor Lamps and Replacements ............... $\quad 22$

Table 6.c HID Lamps - 1000W Mercury Vapor Lamps and Replacements ............. 23

Table 7. Lighting Power Density by Building Type ............................................... $\quad 26$

Table 8. Fixture Shares of Delivered Lumens ......................................................... $\quad 27$

Table 9. Fluorescent System Shäres--1986 Stock ..................................................... 29

Table 10. Fluorescent System Shares--1986 New Construction ............................... 30

Table 11. Fluorescent System Shares--1995 Stock .................................................. 31

Table 12. Standard Fixture Shares of Floor Area--Stock/New Construction ............ $\quad 32$

Table 13. Luminaire Efficiencies ........................................................................... 
Table 14.a. Fluorescent Fixture Saturation as a Percentage of Number of

Lamps per Fixture (1986)

Table 14.b. Fluorescent Fixture Saturation as a Percentage of Number of Lamps per Fixture (1995) ................................................................................... 35

Table 15. Impacts, Costs and Applicability of Controls ........................................ 38

Table 16.a. Heating and Cooling Coincidence Factors for Fast-Foods Restaurant

Table 16.b. Heating and Cooling Coincidence Factors for Hospital ........................ 42

Table 16.c. Heating and Cooling Coincidence Factors for Large Hotel .................. 43

Table 16.d. Heating and Cooling Coincidence Factors for Large Office ................. 43

Table 16.e. Heating and Cooling Coincidence Factors for Large Retail .................. 44

Table 16.f. Heating and Cooling Coincidence Factors for Medium Office ............. 44

Table 16.g. Heating and Cooling Coincidence Factors for Small Hotel/Motel

Table 16.h. Heating and Cooling Coincidence Factors for Sit-Down Restaurant

Table 16.i. Heating and Cooling Coincidence Factors for Supermarket .................. 46

Table 16.j. Heating and Cooling Coincidence Factors for Secondary School .......... 46

\section{List of Figures}

Figure 1. Heating and Cooling Coincidence Factors for Lighting 


\section{INTRODUCTION}

Lighting represents over $20 \%$ of all energy delivered to commercial buildings, and about $40 \%$ of commercial buildings' electricity consumption. Because of this large share in energy consumption, as well as the availability and emergence of efficient lighting technologies, the lighting end use is a major area of attention for energy policy and utility Demand Side Management (DSM) programs.

End-use forecasting models used by utilities and government agencies provide forecasts of energy consumption by fuel type, end use and building type. Energy-efficiency policy analysis and Demand Side Management (DSM) planning, which address specific energy technologies, required the development of new policy analysis tools. Based on these needs, the Electric Power Research Institute has expanded its Commercial End-Use Planning System, COMMEND, to include detailed technology representations of lighting and HVAC. ${ }^{1}$ These detailed technology modules, available in COMMEND 4.0, replaced the generic technology trade-off curves available in previous versions (COMMEND 3.2). LBL assisted in the development of the technology-analysis framework and continues to provide assistance in the development and refinement of technology data for the model.

This report covers all of the building types for the lighting end use and also attempts to characterize the secondary effects of reduction in lighting energy use on HVAC consumption in these building types under different climate conditions. A similar report is available for the HVAC end uses [1]. The HVAC report covers only office buildings at this point, but work is in progress for the other commercial building types.

\section{COMMEND STRUCTURE ${ }^{2}$}

In COMMEND 3.2 and earlier versions of the model, the lighting end use was represented by a single technology tradeoff curve. In COMMEND 4.0, this end-use level of modeling remains available. However, a more detailed option is also available that allows modeling of specific lighting technologies.

The main features of the detailed interior lighting mode $e_{\Perp}$ re as follows:

Lighting Systems. The model deals directly with an enumerated list of specific lighting systems. These systems are defined as specific combinations of lamps, ballasts and fixture types.

Lighting Terminology. In place of general end-use concepts, such as utilization indices, an expanded set of lighting terms are used in the model. Illuminance, or lighting level, is computed in footcandles. Source lumens represent the amount of

1 Heating, ventilation and air conditioning

2 Adapted from COMMEND 4.0 User's Guide, Appendix F [2]. 
light given off by lamps alone. Fixture efficiency and room factors are fractions that convert source lumens to delivered lumens at the work plane. System efficacy defines the amount of light supplied in lumens per Watt of electricity input. Usage is measured directly in hours of use.

Lighting Shares. Lighting systems (lamp/ballast/fixture combinations) are grouped into fixture types. This grouping has advantages for modeling retrofits during the forecast years. Shares are defined for the fixture types and for the systems within each fixture type. Shares give the fraction of lighting capacity that is provided by that system. This system capacity is expressed in delivered lumens per square foot (footcandles), which is a measure of delivered light output, adjusted for lamp/ballast efficiency, fixture efficiency, and room factors.

Decision Models. System shares are computed using a set of decision models. The new construction model gives system shares in new buildings. The lamp replacement model allows lamp changes at the time of lamp decay. The ballast replacement model allows changes in ballast efficiency at the time of ballast decay. The system conversion model allows changes in lamp, ballast, and fixture efficiency, as well as conversion from one light source to another.

\section{DESCRIPTION AND DEVELOPMENT OF DATA}

This report characterizes the present floorstock in terms of lighting technologies and develops cost-efficiency data for these lighting technologies. The report also characterizes the present lighting utilization patterns and lighting level requirements. Lighting/HVAC interactions are also a part of this report. Parameters related to the decision algorithms and characterization of future trends are not a concern of this report. Such parameters are best developed using utility DSM survey data.

Most of the data presented in this report were developed as part of the Lighting Policy Analysis (LPA) [3] at LBL. This report compiles the technology data and presents them in a fashion usable by a forecasting analyst. However, the characterization of lighting/HVAC interactions has been improved and expanded subsequent to the publication of the LPA report.

\section{EQUIPMENT DEFINITIONS}

Lighting systems are defined to be combinations of lamps and ballasts for a given fixture type. The fixture types considered in the data development are: 4-foot fluorescent fixtures, 8-foot fluorescent fixtures, standard (screw-in) fixtures and high-intensity discharge (HID) fixtures. Systems covered in this report either have significant market share or are promising in terms of future utilization. Table 1 shows the system types in the category of fluorescent fixtures, and Table 2 shows system types in the categories of standard (screw-in) and HID fixtures. There are a few system types that have not been 
included in the data set. The most important of these are systems utilizing U-lamps and T10 lamps. Market saturation data are developed for the set of technologies shown in Tables 1 and 2. Cost efficiency data are presented for a broader range of system types.

The properties of the luminaire and controls associated with the lighting system are necessary to complete the characterization of the lighting end-use. Luminaires-sometimes referred to as fixtures--for the fluorescent fixture types considered are (1) lensed troffer, (2) wraparound and (3) parabolic luminaires. Saturation and efficiency data are developed for these three types of fluorescent luminaires. Luminaires considered for the HID fixture type are: (1) round, (2) square, and (3) indirect. Luminaires for the standard fixture type are not enumerated and only average efficiencies are presented for these. In previous work [3], control types considered for conservation analysis were: (1) programmable timers, (2) timers and lumen maintenance, (3) occupancy sensors, and (4) daylighting controls. Energy management systems vary in terms of size and costs, and they mostly perform the above functions. Therefore, they were not explicitly addressed. 
Table 1. Systems with Fluorescent Fixture Types

Fixture Type Ballast Lamp

\section{4-Foot Fluorescent}

Standard Ballast

Standard F40 T12

Reduced Wattage 34WT12

Energy Efficient Magnetic Ballast

Standard F40 T12

Reduced Wattage 34WT12

$\mathrm{T}-8$

Cathode Cutout Ballast

Standard F40 T12

Reduced Wattage 34WT12

T-8

Electronic Ballast

Standard F40 T12

Reduced Wattage 34WT12

T-8

8-Foot Fluorescent

Slimline Lamps

Standard Ballast

Standard F96

Reduced Wattage F96

Energy Efficient Magnetic Ballast

Standard F96

Electronic Ballast

Reduced Wattage F96

Standard F96

Reduced Wattage F96

High Output Lamps

Standard Ballast

Standard F96

Reduced Wattage F96

Energy Efficient Magnetic Ballast

Standard F96

Reduced Wattage F96

Electronic Ballast

Standard F96

Reduced Wattage F96 
Table 2. Systems with Fixtures Types other than Fluorescent

Fixture Type Lamp

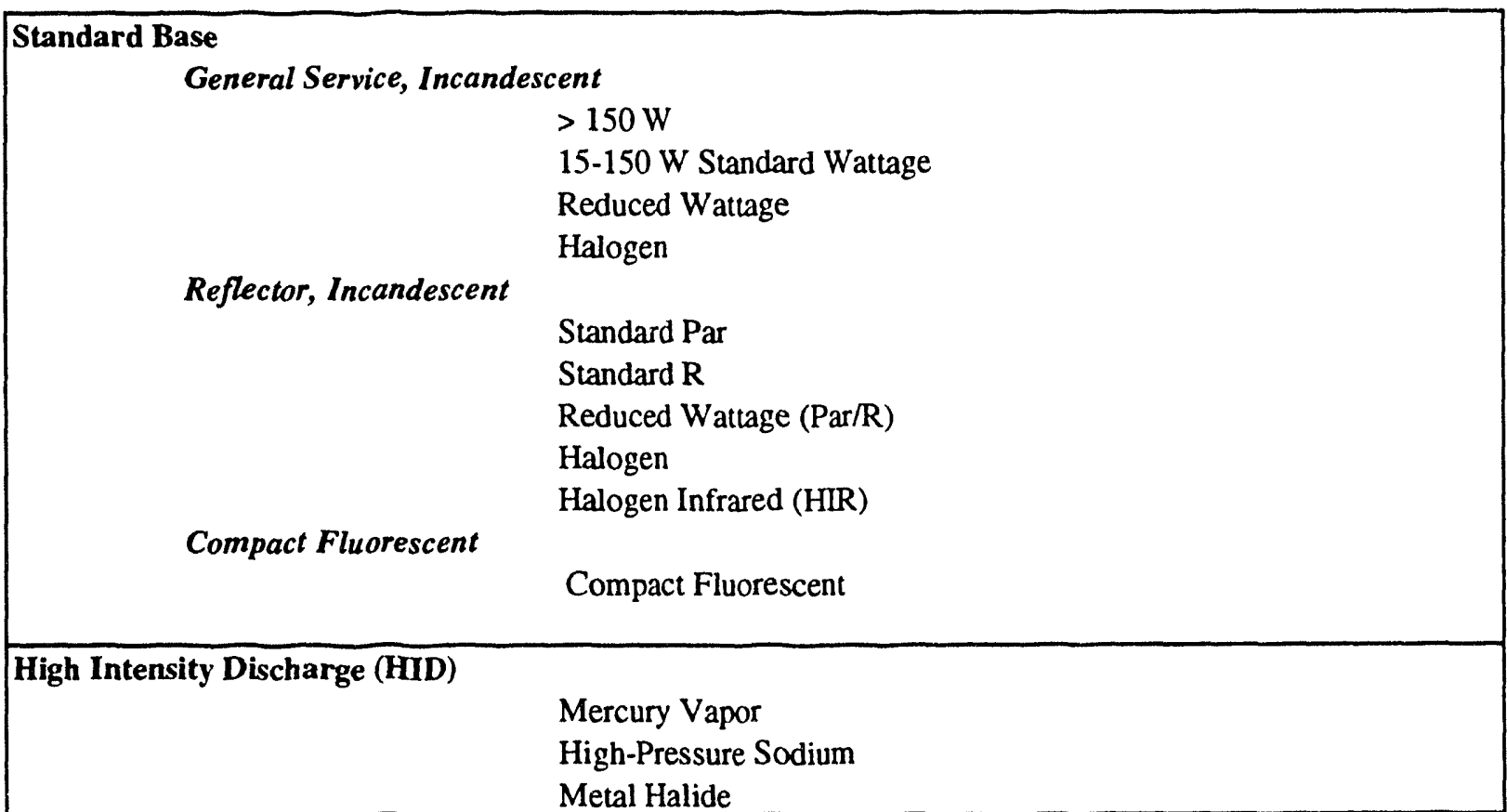




\section{SERVICE AND ELECTRICITY DEMAND}

Service demand is lighting requirements in terms of lighting levels and lighting schedules; and is independent of the lighting technologies utilized. Within the COMMEND 4 framework, operating hours and delivered lighting levels determine the level of service demand. Electricity demand is a function of the service demand, together with other factors like (1) properties and geometry of the space being lit, (2) efficiency of the luminaire used, and (3) efficacy of the lamp/ballast combination.

\section{Service Demand--Operating Hours and Lighting Levels}

Data for lighting operating hours come from CBECS [4] NBECS [5], and the DOE/EIA "Lighting in Commercial Buildings" report [6]. The distinction between building operating hours and lighting operating hours should be made. As an example, DOE/EIA [6] indicates that large office buildings may be operating for 2932 hours annually, where the lighting equipment for the same building type is operated for 3603 effective lighting hours. Effective lighting hours are specified as

$$
\begin{aligned}
& \text { Effective lighting hours }=\quad \text { operating hours }+ \\
& \text { (space lit during non-op. hours/space lit during op. hours) }{ }^{*} \text { non-operating hours. }
\end{aligned}
$$

Table 3 shows annual lighting operating hours by building type. Fluorescent and incandescent operating hours were developed based on communication with EIA. ${ }^{3}$

The distinction between source lumen and delivered lumen requirements should be made. Delivered lumens are measured at the work plane and are independent of the lighting technology used and the characteristics of the room. Level of delivered lumens is obtained as a product of source lumens, luminaire efficiency (fixture efficiency) and the room factor. COMMEND requires levels of delivered lumens by building type. Illuminance-delivered can be estimated using IES Lighting Handbook [7] which gives three levels of recommended lighting intensities for categories of activities. For a given building type, the lighting level has to be developed as the weighted average of these intensities based on the distribution of different activities over the floorstock. EIA has used the higher end of this data to develop average source lumen intensities for CBECS building types [6]. Table 3 shows illuminance requirements by building type derived from the data given in the EIA lighting study [6]. These data have to be multiplied by average luminaire efficiencies and room factors before they can be input to COMMEND.

The building type definitions are not identical in COMMEND and CBECS [4]/EIA [6]. The mapping used in developing data on operating hours and lighting levels by building

${ }^{3}$ Energy Information Administration, U.S. Department of Energy, Washington, D.C. 
type is as follows: ${ }^{4}$

$5 \%$ of Assembly floor area (from CBECS) goes to Colleges (in COMMEND),

95\% of Assembly floor area (from CBECS) goes to Miscellaneous (in COMMEND),

73\% of Education floor area (from CBECS) goes to Schools (in COMMEND), and

$27 \%$ of Education floor area (from CBECS) goes to Colleges (in COMMEND).

\section{Room Factors}

The Room Factor models losses due to factors other than the luminaire, like the room geometry, room surface reflectances, furniture, etc. In the lighting industry, Coefficient of Utilization (CU) is used to quantify the combined effect of luminaire (fixture) efficiency together with room properties. For each luminaire, manufacturers catalogues provide the $\mathrm{CU}$ under various conditions of room geometry and surface properties. Within the COMMEND framework, the effects of the room properties are separated from the luminaire efficiency mainly because the room specifications are independent of the technology that is used to illuminate the space. Luminaire efficiency re presents light output from the luminaire before room factor losses. The product of the room factor for a building type and the average luminaire efficiency for a specific lighting systems utilized in that building type should give the average $\mathrm{CU}$ (e.i weighted average of the CUs for the luminaires used for the specific lighting system, under average conditions for that building type). For commercial buildings, room factors are generally close to 0.6 .

\section{EUI vs. Operating Hours}

In its present state, COMMEND 4 does not utilize the operating hours presented in the input data set. Instead, the Energy Utilization Index (EUI) developed for the lighting end-use determines the level of consumption. Lighting operating hours are part of the output of the program. The operating hours output by the program should be compared to the intended operating hours and model inputs must be corrected to reconcile the two sets. A difference between the two set of operating hours may be an indication of wrong lighting levels and/or market shares of technologies in the input file.

\footnotetext{
${ }^{4}$ Pacific Northwest Laboratories (PNL). Personal communication, Dave Belzer. 1991.
} 
Table 3. Effective Lighting Hours (Annual) and Lighting Levels by Building Type

\begin{tabular}{|lcccc|}
$\begin{array}{l}\text { COMMEND } \\
\text { Building Type }\end{array}$ & $\begin{array}{c}\text { Fluo. } \\
\text { Hours(2) }\end{array}$ & $\begin{array}{c}\text { Incand. } \\
\text { Hours(2) }\end{array}$ & $\begin{array}{c}\text { HID } \\
\text { Hours(2) }\end{array}$ & $\begin{array}{c}\text { Source-Lumen } \\
\text { Level (1) } \\
\text { (lumens/sqft) }\end{array}$ \\
\hline small office & 3624 & 3365 & 3583 & 91 \\
large office & 3624 & 3365 & 3583 & 91 \\
restaurant & 4957 & 5361 & 7223 & 20 \\
retail & 4064 & 3867 & 4883 & 50 \\
grocery & 6019 & 5477 & 8601 & 50 \\
warehouse & 3739 & 3465 & 4711 & 18 \\
school & 2462 & 3337 & 2943 & 100 \\
college & 3249 & 3186 & 3174 & 93 \\
health & 7955 & 8086 & 8694 & 186 \\
lodging & 8572 & 8331 & 8502 & 50 \\
miscellaneous & 4005 & 3062 & 5404 & 64 \\
\hline
\end{tabular}

(1) Source: EIA 1992 [6].

(2) Source: Communication with EIA--based on unpublished information supporting [6]. 


\section{LIGHTING SYSTEMS}

\section{Cost/Efficiency Data for Lighting Systems}

Data presented include service lifetimes, equipment prices and labor costs for lamps and ballasts; and efficacies and watts for lamp/ballast combinations. Tables 4a-g present data for fluorescent lamps and their associated ballasts. The first three of these tables are for 4-foot lamps; operated with energy-efficient magnetic, cathode cutout and electronic ballasts, respectively. Tables $4 \mathrm{~d}-\mathrm{g}$ give data for 8 -foot and 8 -foot-high-output lamps operated with magnetic and electronic ballasts. Tables 5a-c give data for lamps associated with standard (screw-in) systems for general service, reflector type incandescent lamps and compact fluorescent, respectively. Table 6a-c gives the efficient replacements for $100 \mathrm{~W}, 400 \mathrm{~W}$, and $1000 \mathrm{~W}$ Mercury Vapor Lamps, respectively, within the HID fixture type.

Many of the energy-efficient lamp technologies considered in this report are relatively new to the marketplace. In order to arrive at prices that are representative of the price a typical large commercial purchaser would pay for energy-efficient lamps and ballasts, prices have been collected from a wide variety of sources. These sources include wholesalers, manufacturers, distributors, local outlets, and sources of lighting design and analysis software. There is no single accepted consensus on prices for these products. Nevertheless, input from all of the above sources contributes to the development of the prices given in this section. The LPA report [3] gives the methodology for developing prices presented in this report from the above-mentioned sources [3]. Both equipment price data and labor rates are 1992 prices in 1990 dollars.

Service lifetimes of lamps and ballast are presented in years instead of hours of use for the convenience of the reader. Each table indicates the annual operating hours used for that table. Since operating hours vary by building type, the service lifetime figures should be used with caution. COMMEND input requires lifetimes in hours of operation.

The lamp/ballast efficacy and watts are corrected for ballast factor and also for thermal interactions. The correction for thermal interactions is applied only on 4-foot fluorescent technologies based on saturation of different luminaire types over the stock of 4-foot lamps. It is assumed that thermal interactions in 8-foot fixtures are negligible because of the high saturation of open fixtures.

The most popular high-efficiency fluorescent lamp/ballast combination on the market is the T8 lamp with electronic ballast. The standard-wattage fluorescent lamps in all categories will become obsolete under the standards of the Energy Policy Act of 1992 (EPAct) in late 1995. 
For incandescent reflector lamps, the standard- and reduced-wattage lamps will become obsolete at the same time under EPAct. The halogen PAR lamp and the halogen infrared PAR will become the efficient substitutes. The EPAct does not impose standards on incandescent general service lamps. In this family, the halogen lamp may become more popular if its price drops. Lamp companies may introduce a halogen IR lamp. Research on various filament applications may result in even higher efficacies.

Compact fluorescent lamps are moving towards models with electronic ballasts. Dimmable ballasts allow ther: use in more applications.

The high intensity discharge (HID) lamp market is moving away from mercury vapor to the more efficient metal halide and high pressure sodium. Lower wattage versions with better color rendering will allow wider application of these sources. 
Table 4a. Characteristics of 4-Foot Fluorescent Lamps with Energy-Efricient Magnetic Ballast

( 2 lamps, 1 ballast per fixture)

\begin{tabular}{|c|c|c|c|c|c|c|c|c|}
\hline No. & Technology Option & $\begin{array}{l}\text { Ballast } \\
\text { Labor } \\
\text { Cost }(1) \\
(\$ 1990) \\
\end{array}$ & $\begin{array}{c}\text { Ballast } \\
\text { Price } \\
(\$ 1990) \\
\end{array}$ & $\begin{array}{l}\text { Ballast } \\
\text { Service } \\
\text { Life (2) } \\
\text { (years) } \\
\end{array}$ & $\begin{array}{l}\text { ANSI } \\
\text { Watts }\end{array}$ & $\begin{array}{c}\text { Rated } \\
\text { Lumens } \\
\text { (lum/lamp) }\end{array}$ & $\begin{array}{c}\text { Fixture } \\
\text { Watts (3) } \\
\end{array}$ & $\begin{array}{c}\text { Efficacy(4) } \\
\text { (lum/watt) }\end{array}$ \\
\hline $0 *$ & Baseline (F40CW T12, 40 W cool white) & 24.93 & 13.61 & 12.00 & 88.0 & 3,050 & 78.9 & 69.6 \\
\hline $1 *$ & F40CW/WM (34W, reduced wattage) & 24.93 & 13.61 & 12.00 & 72.0 & 2,650 & 73.7 & 60.4 \\
\hline 2 & F40SP41/WM (34W, RE 70) & 24.93 & 13.61 & 12.00 & 72.0 & 2,800 & 73.7 & 63.8 \\
\hline 3 & F40AXT10 (1 1/4" dia) & 24.93 & 13.61 & 12.00 & 92.0 & 3,700 & 82.9 & 80.3 \\
\hline $4^{*}$ & F32T8 (1" dia, triphosphor) & 24.93 & 13.88 & 12.00 & 70.0 & 2,900 & 64.0 & 82.5 \\
\hline 5 & F40SP41 (40W, RE 70) & 24.93 & 13.61 & 12.00 & 88.0 & 3,200 & 78.9 & 73.0 \\
\hline 6 & F40SP41/WMP (cathode cutout lamp, RE 70) & 24.93 & 13.61 & 12.00 & 64.4 & 2,650 & 62.0 & 76.9 \\
\hline 7 & F40SPX41 (40W, RE 80) & 24.93 & 13.61 & 12.00 & 88.0 & 3,250 & 78.9 & 74.1 \\
\hline 8 & F40CW/WMP (cathode cutout lamp) & 24.93 & 13.61 & 12.00 & 64.3 & 2,525 & 62.0 & 73.3 \\
\hline 9 & F40SPX41/WM (34W, RE 80) & 24.93 & 13.61 & 12.00 & 72.0 & 2,850 & 73.7 & 65.0 \\
\hline
\end{tabular}

• Lamp Replacement Cost:

Lamp Replacement Cost:
\begin{tabular}{|clccc|} 
No. & Technology Option & $\begin{array}{c}\text { Lamp } \\
\text { Labor } \\
\text { Cost }(1) \\
(\$ 1990)\end{array}$ & $\begin{array}{c}\text { Lamp } \\
\text { Price } \\
(\$ 1990)\end{array}$ & $\begin{array}{c}\text { Lamp } \\
\text { Service } \\
\text { Life }(2) \\
\text { (years) }\end{array}$ \\
\hline $0^{*}$ & Baseline (F40CW T12, 40 W cool white) & 8.54 & 2.24 & 3.41 \\
$1^{*}$ & F40CW/WM (34W, reduced wattage) & 8.54 & 3.12 & 3.41 \\
2 & F40SP41/WM (34W, RE 70) & 8.54 & 6.08 & 3.41 \\
3 & F40AXT10 (1 1/4" dia) & 8.54 & 11.20 & 4.09 \\
$4^{*}$ & F32T8 (1" dia, triphosphor) & 8.54 & 4.90 & 3.66 \\
5 & F40SP41 (40W, RE 70) & 8.54 & 5.78 & 3.41 \\
6 & F40SP41/WMP (cathode cutout lamp, RE 70) & 8.54 & 7.36 & 3.41 \\
7 & F40SPX41 (40W, RE 80) & 8.54 & 11.78 & 3.41 \\
8 & F40CW/WMP (cathode cutout lamp) & 8.54 & 4.76 & 3.41 \\
9 & F40SPX41/WM (34W, RE 80) & 8.54 & 12.28 & 3.41 \\
\hline
\end{tabular}

* Appears in Table 1.

(1) Labor Rates $(\$ 1990$ per hour) $=\$ 25.63$ (electrician helper), $\$ 36.69$ (electrician)--it is assumed that the time for lamp replacement is charged at electrician helper rate, and the time for ballast replacement is evenly split between the electrician and helper.

(2) Calculated using annual lighting hours of 4,103 .

(3) Corrected for fixture interactions.

(4) Corrected for fixture interactions and ballast factor 
Table 4b. Characteristics of 4-Foot Fluorescent Lamps with Cathode-Cutout Ballast (2 lamps, 1 ballast per fixture)

\begin{tabular}{|c|c|c|c|c|c|c|c|c|}
\hline No. & Technology Option & $\begin{array}{l}\text { Ballast } \\
\text { Labor } \\
\text { Cost }(1) \\
(\$ 1990) \\
\end{array}$ & $\begin{array}{c}\text { Ballast } \\
\text { Price } \\
(\$ 1990) \\
\end{array}$ & $\begin{array}{l}\text { Ballast } \\
\text { Service } \\
\text { Life (2) } \\
\text { (years) } \\
\end{array}$ & $\begin{array}{l}\text { ANSI } \\
\text { Watts }\end{array}$ & $\begin{array}{c}\text { Rated } \\
\text { Lumens } \\
\text { (lum/lamp) }\end{array}$ & $\begin{array}{c}\text { Fixture } \\
\text { Watts (3) }\end{array}$ & $\begin{array}{c}\text { Efficacy(4) } \\
\text { (lum/watt) }\end{array}$ \\
\hline $0^{*}$ & Baseline (F40CW T12, 40W cool white) & 26.52 & 18.52 & 12.00 & 80.0 & 3,050 & 71.5 & 77.6 \\
\hline 1 & F40AXT10 (1 1/4" dia) & 26.52 & 18.52 & 12.00 & 88.0 & 3,700 & 75.5 & 89.2 \\
\hline 2 & F40SPX41/WM (34W, RE 80) & 26.52 & 18.52 & 12.00 & 66.0 & 2,850 & 67.5 & 71.8 \\
\hline 3 & F40SP41/WM (34W, RE 70) & 26.52 & 18.52 & 12.00 & 66.0 & 2,800 & 67.5 & 70.5 \\
\hline $4^{*}$ & F40CW/WM (34W, reduced wattage) & 26.52 & 18.52 & 12.00 & 66.0 & 2,650 & 67.5 & 66.7 \\
\hline 5 & F40SP41 (40W, RE 70) & 26.52 & 18.52 & 12.00 & 80.0 & 3,200 & 71.5 & 81.5 \\
\hline $6^{*}$ & F32T8 (1" dia, triphosphor) & 26.52 & 18.52 & 12.00 & 62.0 & 2,900 & 65.1 & 82.0 \\
\hline 7 & F40SPX41 (40W, RE 80) & 26.52 & 18.52 & 12.00 & 80.0 & 3,250 & 71.5 & 82.7 \\
\hline \multicolumn{2}{|c|}{ Lamp Replacement Cost: } & Lamp & & Lamp & & & & \\
\hline & & Labor & Lamp & Service & & & & \\
\hline & & Cost (1) & Price & Life (2) & & & & \\
\hline$\frac{\text { No. }}{0^{*}}$ & Technology Option & $(\$ 1990)$ & $(\$ 1990)$ & (years) & & & & \\
\hline $0^{*}$ & Baseline (F40CW T12, 40W cool white) & 8.54 & 2.24 & 3.41 & & & & \\
\hline 1 & F40AXT10 (1 $1 / 4^{\prime \prime ~ d i a) ~}$ & 8.54 & 11.20 & 4.09 & & & & \\
\hline 2 & F40SPX41/WM (34W, RE 80) & 8.54 & 12.28 & 3.41 & & & & \\
\hline 3 & F40SP41/WM (34W, RE 70) & 8.54 & 6.08 & 3.41 & & & & \\
\hline $4^{*}$ & F40CW/WM ( $34 \mathrm{~W}$, reduced wattage) & 8.54 & 3.12 & 3.41 & & & & \\
\hline 5 & F40SP41 (40W, RE 70) & 8.54 & 5.78 & 3.41 & & & & \\
\hline $6^{*}$ & F32T8 (1" dia, triphosphor) & 8.54 & 4.90 & 3.66 & & & & \\
\hline 7 & F40SPX41 (40W, RE 80) & 8.54 & 11.78 & 3.41 & & & & \\
\hline
\end{tabular}

* Appears in Table 1 .

lamp replacement is charged at electrician helper rate, and the time for ballast replacement is evenly split between the electrician and helper.

(2) Calculated using annual lighting hours of 4,103.

(3) Corrected for fixture interactions.

(4) Corrected for fixture interactions and ballast factor 
Table 4c. Characteristics of 4-Foot Fluorescent Lamps with Electronic Ballast

( 2 lamps, 1 ballast per fixture)

\begin{tabular}{|c|c|c|c|c|c|c|c|c|}
\hline No. & Technology Option & $\begin{array}{l}\text { Ballast } \\
\text { Labor } \\
\text { Cost (1) } \\
(\$ 1990) \\
\end{array}$ & 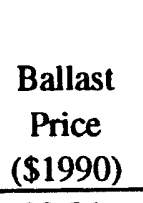 & $\begin{array}{l}\text { Ballast } \\
\text { Service } \\
\text { Life (2) } \\
\text { (years) } \\
\end{array}$ & $\begin{array}{l}\text { ANSI } \\
\text { Watts } \\
\end{array}$ & $\begin{array}{c}\text { Rated } \\
\text { Lumens } \\
\text { (lum/lamp) }\end{array}$ & $\begin{array}{c}\text { Fixture } \\
\text { Watts(3) } \\
\end{array}$ & $\begin{array}{r}\text { Efficacy(4) } \\
\text { (lum/watt) }\end{array}$ \\
\hline $0^{*}$ & Baseline (F40CW T12, 40W cool white) & 26.52 & 22.56 & 12.00 & 72.0 & 3,050 & 66.2 & 78.3 \\
\hline 1 & F40AXT10 (1 1/4" dia $)$ & 26.52 & 22.56 & 12.00 & 83.0 & 3,700 & 70.2 & 89.6 \\
\hline 2 & F32T8 (1" dia, triphosphor) w/Rapid Start & 26.52 & 21.76 & 12.00 & 62.0 & 2,900 & 55.9 & 88.2 \\
\hline 3 & F40SP41 (40W, RE 70) & 26.52 & 22.56 & 12.00 & 72.0 & 3,200 & 66.2 & 82.2 \\
\hline 4 & F40SPX41 (40W, RE 80) & 26.52 & 22.56 & 12.00 & 72.0 & 3,250 & 66.2 & 83.5 \\
\hline $5^{*}$ & F32T8 (1" dia, triphosphor) w/Instant Start & 26.52 & 25.44 & 12.00 & 63.0 & 2,900 & 52.7 & 101.3 \\
\hline 6 & F40SPX41/WM (34W, RE 80) & 26.52 & 22.56 & 12.00 & 60.0 & 2,850 & 60.1 & 78.7 \\
\hline 7 & F40SP41/WM (34W, RE 70) & 26.52 & 22.56 & 12.00 & 60.0 & 2,800 & 60.1 & 77.3 \\
\hline $8^{*}$ & F40CW/WM ( $34 \mathrm{~W}$, reduced wattage) & 26.52 & 22.56 & 12.00 & 60.0 & 2,650 & 60.1 & 73.2 \\
\hline
\end{tabular}

$\ddot{\omega}$

Lamp Replacement Cost:
\begin{tabular}{|clccc|} 
No. & Technology Option & $\begin{array}{c}\text { Lamp } \\
\text { Labor } \\
\text { Cost }(1) \\
(\$ 1990)\end{array}$ & $\begin{array}{c}\text { Lamp } \\
\text { Price } \\
(\$ 1990)\end{array}$ & $\begin{array}{c}\text { Lamp } \\
\text { Service } \\
\text { Life }(2) \\
\text { (years) }\end{array}$ \\
\hline $0^{*}$ & Baseline (F40CW T12, 40W cool white) & 8.54 & 2.24 & 3.41 \\
1 & F40AXT10 (1 1/4" dia) & 8.54 & 11.20 & 4.09 \\
2 & F32T8 (1" dia, triphosphor) w/Rapid Start & 8.54 & 4.90 & 3.66 \\
3 & F40SP41 (40W, RE 70) & 8.54 & 5.78 & 3.41 \\
4 & F40SPX41 (40W, RE 80) & 8.54 & 11.78 & 3.41 \\
$5^{*}$ & F32T8 (1" dia, triphosphor) w/Instant Start & 8.54 & 4.90 & 2.74 \\
6 & F40SPX41/WM (34W, RE 80) & 8.54 & 12.28 & 3.41 \\
7 & F40SP41/WM (34W, RE 70) & 8.54 & 6.08 & 3.41 \\
$8^{*}$ & F40CW/WM (34W, reduced wattage) & 8.54 & 3.12 & 3.41 \\
\hline
\end{tabular}

* Appears in Table 1.

(1) Labor Rates $(\$ 1990$ per hour) $=\$ 25.63$ (electrician helper), $\$ 36.69$ (electrician)--it is assumed that the time for

lamp replacement is charged at electrician helper rate, and the time for ballast replacement is evenly split between the electrician and helper.

(2) Calculated using annual lighting hours of 4,103.

(3) Corrected for fixture interactions.

(4) Corrected for fixture interactions and ballast facto:- 
Table 4d. Characteristics of 8-Foot Fluorescent Lamps with Energy-Efncient Magnetic Ballast

(2 lamps , 1 ballast per fixture)

\begin{tabular}{|c|c|c|c|c|c|c|c|}
\hline No. & Technology Option & $\begin{array}{c}\text { Ballast } \\
\text { Labor } \\
\text { Cost }(1) \\
(\$ 1990) \\
\end{array}$ & $\begin{array}{l}\text { Ballast } \\
\text { Price } \\
(\$ 1990) \\
\end{array}$ & $\begin{array}{l}\text { Ballast } \\
\text { Service } \\
\text { Life (2) } \\
\text { (years) }\end{array}$ & $\begin{array}{l}\text { ANSI } \\
\text { Watts } \\
1500\end{array}$ & $\begin{array}{c}\text { Rated } \\
\text { Lumens } \\
\text { (lum/lamp) }\end{array}$ & $\begin{array}{c}\begin{array}{c}\text { Efficacy(3) } \\
\text { (lum/watt) }\end{array} \\
701\end{array}$ \\
\hline $0^{*}$ & Baseline (F96CW T12, $75 \mathrm{~W}$ cool white & 31.16 & 21.50 & 12.00 & 158.0 & 6,150 & 70.1 \\
\hline $1 *$ & F96T12/CW/WM (60W, reduced wattage) & 31.16 & 21.50 & 12.00 & 136.0 & 5,500 & 72.8 \\
\hline 2 & F96T12/SPX41/WM (60W, RE 80) & 31.16 & 21.50 & 12.00 & 136.0 & 5,900 & 78.1 \\
\hline 3 & F96T12/SP41/WM (60W, RE 70) & 31.16 & 21.50 & 12.00 & 136.0 & 5,750 & 76.1 \\
\hline 4 & F96T12/SP41 (75W, RE 70) & 31.16 & 21.50 & 12.00 & 158.0 & 6,425 & 73.2 \\
\hline 5 & F96T12/SPX41 (75W, RE 80) & 31.16 & 21.50 & 12.00 & 158.0 & 6,550 & 74.6 \\
\hline
\end{tabular}

Lamp Replacement Cost:

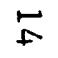

\begin{tabular}{|clccc|}
\multicolumn{1}{l}{ No. } & Technology Option & $\begin{array}{c}\text { Cost }(1) \\
(\$ 1990)\end{array}$ & $\begin{array}{c}\text { Price } \\
(\$ 1990)\end{array}$ & $\begin{array}{c}\text { Life }(2) \\
\text { (years) }\end{array}$ \\
\hline $0^{*}$ & Baseline (F96CW T12, 75 W cool white & 6.19 & 5.20 & 2.05 \\
$1^{*}$ & F96T12/CW/WM (60W, reduced wattage) & 6.19 & 6.54 & 2.05 \\
2 & F96T12/SPX41/WM (60W, RE 80) & 6.19 & 23.32 & 2.05 \\
3 & F96T12/SP41/WM (60W, RE 70) & 6.19 & 13.32 & 2.05 \\
4 & F96T12/SP41 (75W, RE 70) & 6.19 & 13.74 & 2.05 \\
5 & F96T12/SPX41 (75W, RE 80) & 6.19 & 22.46 & 2.05 \\
\hline
\end{tabular}

* Appears in Table 1.

(1) Labor Rates ( $\$ 1990$ per hour) $=\$ 25.63$ (electrician helper), $\$ 36.69$ (electrician)--it is assumed that the time for lamp replacement is charged at electrician helper rate, and the time for ballast replacement is evenly split between the electrician and helper.

(2) Calculated using annual lighting hours of 4,103 .

(3) Corrected for ballast factor. 
Table 4e. Characteristics of 8-Foot Fluorescent Hight-Output Lamps with Energy-Efricient Magnetic Ballast (2 lamps, 1 ballast per fixture)

\begin{tabular}{|c|c|c|c|c|c|c|c|}
\hline No. & Technology Option & $\begin{array}{c}\text { Ballast } \\
\text { Labor } \\
\text { Cost }(1) \\
(\$ 1990) \\
\end{array}$ & $\begin{array}{c}\text { Ballast } \\
\text { Price } \\
(\$ 1990) \\
\end{array}$ & $\begin{array}{l}\text { Ballast } \\
\text { Service } \\
\text { Life (2) } \\
\text { (years) } \\
\end{array}$ & $\begin{array}{l}\text { ANSI } \\
\text { Watts } \\
2270\end{array}$ & $\begin{array}{c}\text { Rated } \\
\text { Lumens } \\
\text { (lum/lamp) }\end{array}$ & $\begin{array}{c}\text { Efficacy(3) } \\
\text { (lum/watt) }\end{array}$ \\
\hline $0^{*}$ & Baseline (F96T12/CW/HO, $110 \mathrm{~W}$ cool white) & 31.16 & 36.12 & 12.00 & 237.0 & 8,900 & 67.6 \\
\hline 1 & F96T12/SP41/HO/WM (95W, RE 70) & 31.16 & 36.12 & 12.00 & 209.6 & 8,350 & 71.9 \\
\hline 2 & F96T12/SP41/HO (110W, RE 70) & 31.16 & 36.12 & 12.00 & 237.0 & 9,200 & 69.9 \\
\hline $3 *$ & F96T12/CW/HO/WM (95W reduced wattage) & 31.16 & 36.12 & 12.00 & 209.0 & 8,000 & 68.9 \\
\hline \multirow{3}{*}{\multicolumn{2}{|c|}{ Lamp Replacement Cost: }} & Lamp & & Lamp & & & \\
\hline & & Labor & Lamp & Service & & & \\
\hline & & Cost (1) & $\begin{array}{l}\text { Price } \\
\text { (\$1990) }\end{array}$ & $\begin{array}{l}\text { Life (2) } \\
\text { (vears) }\end{array}$ & & & \\
\hline $0^{*}$ & Baseline (F96T12/CW/HO, $110 \mathrm{~W}$ cool white) & 6.62 & 7.04 & & & & \\
\hline 1 & F96T12/SP41/HO/WM (95W, RE 70) & 6.62 & 15.00 & 2.05 & & & \\
\hline 2 & F96T12/SP41/HO (110W, RE 70) & 6.62 & 14.72 & 2.05 & & & \\
\hline 3* & F96T12/CW/HO/WM (95W reduced wattage) & 6.62 & 9.00 & 2.05 & & & \\
\hline
\end{tabular}

* Appears in Table 1.

(1) Labor Rates ( $\$ 1990$ per hour) $=\$ 25.63$ (electrician helper), $\$ 36.69$ (electrician)--it is assumed that the time for

lamp replacement is charged at electrician helper rate, and the time for ballast replacement is evenly split between the electrician and helper.

(2) Calculated using annual lighting hours of 4,103.

(3) Corrected for ballast factor. 
Table 4f. Characteristics of 8-Foot Fluorescent Lamps with Electronic Ballast

(2 lamps, 1 ballast per fixture)

\begin{tabular}{|c|c|c|c|c|c|c|c|}
\hline No. & Technology Option & $\begin{array}{l}\text { Ballast } \\
\text { Labor } \\
\text { Cost }(1) \\
(\$ 1990) \\
\end{array}$ & $\begin{array}{l}\text { Ballast } \\
\text { Price } \\
(\$ 1990) \\
\end{array}$ & $\begin{array}{l}\text { Ballast } \\
\text { Service } \\
\text { Life (2) } \\
\text { (years) } \\
\end{array}$ & $\begin{array}{l}\text { ANSI } \\
\text { Watts } \\
\end{array}$ & $\begin{array}{c}\text { Rated } \\
\text { Lumens } \\
\text { (lum/lamp) }\end{array}$ & $\begin{array}{c}\text { Efficacy(3) } \\
\text { (lum/watt) }\end{array}$ \\
\hline $0^{*}$ & Baseline (F96CW T12, 75 W cool white) & 31.16 & 29.20 & 12.00 & 132.0 & 6,150 & 83.9 \\
\hline $1^{*}$ & F96T12/WM (60W, reduced wattage) & 31.16 & 29.20 & 12.00 & 110.0 & 5,500 & 90.0 \\
\hline 2 & F96T12/SP41/WM (60W, RE 70) & 31.16 & 29.20 & 12.00 & 110.0 & 5,750 & 94.1 \\
\hline 3 & F96T12/SPX41/WM (60W, RE 80) & 31.16 & 29.20 & 12.00 & 110.0 & 5,900 & 96.5 \\
\hline 4 & F96T12/SP41 (75W, RE 70) & 31.16 & 29.20 & 12.00 & 132.0 & 6,425 & 87.6 \\
\hline 5 & F96T12/SPX41 (75W, RE 80) & 31.16 & 29.20 & 12.00 & 132.0 & 6,550 & 89.3 \\
\hline 6 & F096T $8 / 41 \mathrm{~K}$ & 31.16 & 37.50 & 12.00 & 105.0 & 5,800 & 93.9 \\
\hline \multirow{3}{*}{\multicolumn{2}{|c|}{ Lamp Replacement Cost: }} & Lamp & & Lamp & & & \\
\hline & & Labor & Lamp & Service & & & \\
\hline & & $\begin{array}{l}\text { Cost }(1) \\
(\$ 1990)\end{array}$ & $\begin{array}{c}\text { Price } \\
(\$ 1990)\end{array}$ & $\begin{array}{c}\text { Life }(2) \\
\text { (years) }\end{array}$ & & & \\
\hline $0^{*}$ & Baseline (F96CW T12, 75 W cool white) & 6.19 & 5.20 & 2.05 & & & \\
\hline $1^{*}$ & F96T12/WM (60W, reduced wattage) & 6.19 & 6.54 & 2.05 & & & \\
\hline 2 & F96T12/SP41/WM (60W, RE 70) & 6.19 & 13.32 & 2.05 & & & \\
\hline 3 & F96T12/SPX41/WM (60W, RE 80) & 6.19 & 23.32 & 2.05 & & & \\
\hline 4 & F96T12/SP41 (75W, RE 70) & 6.19 & 13.74 & 2.05 & & & \\
\hline 5 & F96T12/SPX 41 (75W, RE 80) & 6.19 & 22.46 & 2.05 & & & \\
\hline 6 & F096T8/41K & 6.19 & 17.00 & 2.56 & & & \\
\hline
\end{tabular}

* Appears in Table 1.

(1) Labor Rates $(\$ 1990$ per hour) $=\$ 25.63$ (electrician helper), $\$ 36.69$ (electrician)--it is assumed that the time for

lamp replacement is charged at electrician helper rate, and the time for ballast replacement is evenly split between the electrician and helper.

(2) Calculated using annual lighting hours of 4,103 .

(3) Corrected for ballast factor. 
Table 4g. Characteristics of 8-Foot Fluorescent High-Output Lamps with Electronic Ballast

(2 lamps, 1 ballast per fixture)

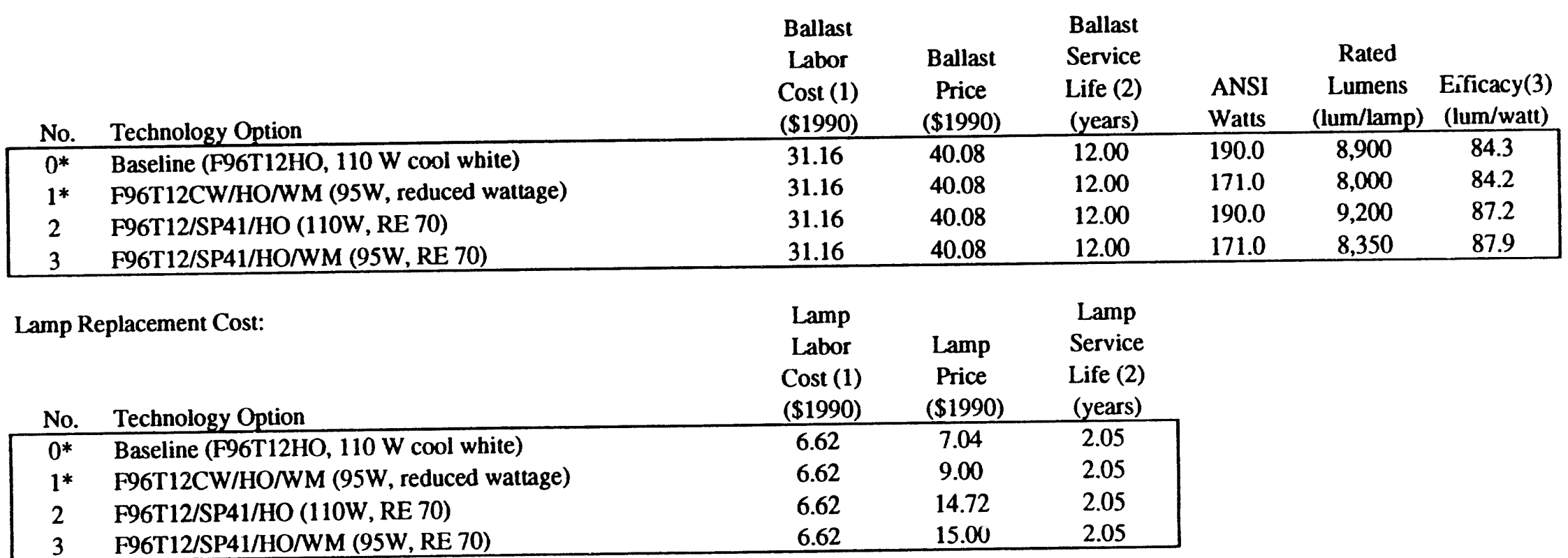

* Appears in Table 1.

(1) Labor Rates ( $\$ 1990$ per hour) $=\$ 25.63$ (electrician helper), $\$ 36.69$ (electrician)--it is assumed that the time for

lamp replacement is charged at electrician helper rate, and the time for ballast replacement is evenly split between the electrician and helper.

(2) Calculated using annual lighting hours of 4,103 .

(3) Corrected for ballast factor. 
Table 5a. Characteristics of General Service Incandescent Lamps

\begin{tabular}{|c|c|c|c|c|c|c|c|}
\hline No. & Technology Option & $\begin{array}{c}\text { Lamp } \\
\text { Labor } \\
\text { Cost }(1) \\
(\$ 1990) \\
\end{array}$ & $\begin{array}{c}\text { Lamp } \\
\text { Price } \\
(\$ 1990) \\
\end{array}$ & $\begin{array}{c}\text { Lamp } \\
\text { Service } \\
\text { Life (2) } \\
\text { (years) }\end{array}$ & Watts & $\begin{array}{c}\text { Rated } \\
\text { Lumens }\end{array}$ & $\begin{array}{c}\text { Efficacy } \\
\text { (lum/Watt) }\end{array}$ \\
\hline $0^{*}$ & Baseline 75A (75W) & 2.14 & 0.34 & 0.18 & 75 & 1,190 & 15.9 \\
\hline $1 *$ & $75 \mathrm{~A} / 67 \mathrm{WM}$ (67W reduced wattage) & 2.14 & 0.45 & 0.18 & 67 & 1,130 & 16.9 \\
\hline $2 *$ & $72 \mathrm{~W}$ halogen & 2.14 & 1.87 & 0.82 & 72 & 1,300 & 18.1 \\
\hline 3 & $70 \mathrm{~A} / \mathrm{ML} / \mathrm{LL}$ (70W reduced wattage) & 2.14 & 0.62 & 0.18 & 70 & 1,140 & 16.3 \\
\hline
\end{tabular}

* Appears in Table 2.

(1) Labor Rates ( $\$ 1990$ per hour) $=\$ 25.63$ (electrician helper), $\$ 36.69$ (electrician)--it is assumed that the time for lamp replacement is charged at electrician helper rate.

(2) Calculated using annual lighting hours of 4,270. 
Table 5b. Characteristics of Reflector Type Incandescent Lamps

\begin{tabular}{|llcccccc|}
\multicolumn{1}{c}{} & $\begin{array}{c}\text { Lamp } \\
\text { Labor } \\
\text { No. }\end{array}$ & $\begin{array}{c}\text { Lamp } \\
\text { Cost }(1) \\
(\$ 1990)\end{array}$ & $\begin{array}{c}\text { Lamp } \\
\text { Price } \\
(\$ 199)\end{array}$ & $\begin{array}{c}\text { Service } \\
\text { Life }(2) \\
\text { (years) }\end{array}$ & Watts & $\begin{array}{c}\text { Rated } \\
\text { Lumens }\end{array}$ & $\begin{array}{c}\text { Efficacy } \\
\text { (lum/Watt) }\end{array}$ \\
\hline $0^{*}$ & Baseline 150PAR/FL & 2.14 & 2.66 & 0.47 & 150 & 2,000 & 13.3 \\
$1^{*}$ & 15OPAR/FL/120WM (120W red watt) & 2.14 & 3.63 & 0.47 & 120 & 1,500 & 12.5 \\
$2^{*}$ & 90PAR/FL/HAL (90W halogen) & 2.14 & 4.91 & 0.47 & 90 & n/a & $20(3)$ \\
$3^{*}$ & 60PAR/HIR (60W halogen infrared) & 2.14 & 6.15 & 0.59 & 60 & n/a & $30(3)$ \\
\hline
\end{tabular}

* Appears in Table 2.

(1) Labor Rates ( $\$ 1990$ per hour) $=\$ 25.63$ (electrician helper), $\$ 36.69$ (electrician)--it is assumed that the time for lamp replacement is charged at electrician helper rate.

(2) Calculated using annual lighting hours of 4,270.

(3) Estimate. Source: Eley Associates. 1993. Advanced Lighting Guidelines: 1993, pp. 7-3. Eley Associates, San Francisco, CA.

n/a Data on lumen output not available. 
Table 5c. Characteristics of Compact Fluorescent Lamps

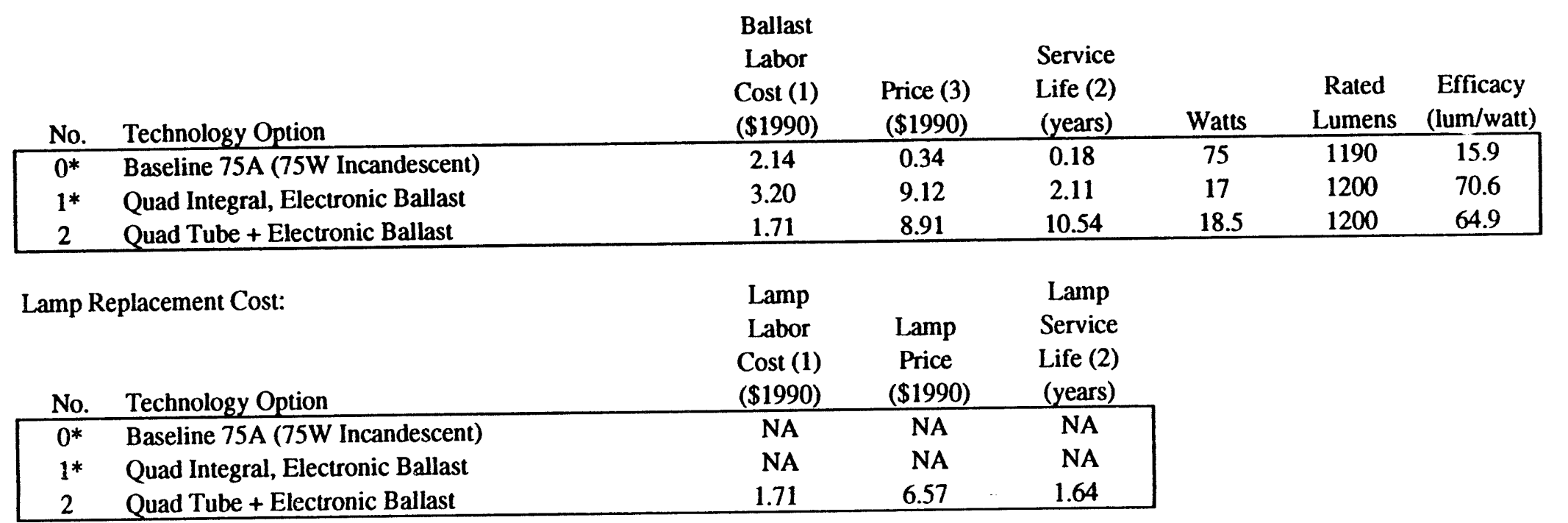

\footnotetext{
* Appears in Table 2 .
}

(1) Labor Rates $(\$ 1990$ per hour) $=\$ 25.63$ (electrician helper $)$

(2) Calculated using annual labor hours of 4,270.

(3) For equipment purchased and replaced as a unit (Options 0 and 1), Price is unit cost (for $0=$ lamp, for $1=$ lamp/ballast) and is

incurred once every Service Life. For equipment with two sections that are replaced at different intervals (Option 2), Price is

the ballast cost and is incurred every Service Life.

NA Not Applicable 
Table 6a. HID Lamps - 100W Mercury Vapor Lamps and Replacements

\begin{tabular}{|c|c|c|c|c|c|c|c|c|}
\hline No. & Technology Option & Base(1) & $\begin{array}{l}\text { Ballast } \\
\text { Labor } \\
\text { Cost }(2) \\
(\$ 1990) \\
\end{array}$ & $\begin{array}{c}\text { Ballast } \\
\text { Price } \\
(\$ 1990) \\
\end{array}$ & $\begin{array}{l}\text { Ballast } \\
\text { Service } \\
\text { Life (3) } \\
\text { (years) } \\
\end{array}$ & $\begin{array}{c}\text { System } \\
\text { Watts }\end{array}$ & $\begin{array}{c}\text { Lamp } \\
\text { Rated } \\
\text { Lumens } \\
\text { (Initial) }\end{array}$ & $\begin{array}{c}\text { Efficacy } \\
\text { (lum/watt) }\end{array}$ \\
\hline 0 & Baseline (Mercury Vapor, $100 \mathrm{~W}$ H38JA-100/DX) & mogul & 32.72 & 31.00 & 18.31 & 118.0 & 4,200 & 35.6 \\
\hline 1 & LU50 (HPS 50W, S68MS-50) (low CRI) & mogul & 32.72 & 84.58 & 18.31 & 66.0 & 4,000 & 60.6 \\
\hline 2 & LU70/DX/MED (HPS 70W, S62LG-70/DX)(CRI 65) & medium & 32.72 & 72.60 & 18.31 & 91.0 & 3,800 & 41.8 \\
\hline
\end{tabular}

Lamp Replacement Cost:

\begin{tabular}{|clrrrr|}
\hline & & & & \\
Lamp Replacement Cost: & $\begin{array}{c}\text { Lamp } \\
\text { Labor }\end{array}$ & $\begin{array}{c}\text { Lamp } \\
\text { Service }\end{array}$ \\
& & $\begin{array}{c}\text { Cost }(2) \\
\text { Price }\end{array}$ & $\begin{array}{c}\text { Life (3) } \\
\text { (years) }\end{array}$ \\
No. & Technology Option & $(\$ 1990)$ & $(\$ 1990)$ & 1.68 & 4.88 \\
0 & Baseline (Mercury Vapor, 100 W H38JA-100/DX) & 7.26 & 15.68 & 4.88 \\
1 & LU50 (HPS 50W, S68MS-50) (low CRI) & 7.26 & 27.40 & 4.05 \\
\hline
\end{tabular}

(1) Medium is a $27 \mathrm{~mm}(11 / 16 ")$ screw in base and mogul is a $40 \mathrm{~mm}$ (1 19/32") screw in base.

(2) Labor Rates ( $\$ 1990$ per hour) $=\$ 25.63$ (electrician helper), $\$ 36.69$ (electrician)--it is assumed that the time for

lamp replacement is charged at electrician helper rate, and the time for ballast replacement is evenly split between the electrician and helper.

(3) Calculated using annual lighting hours of 4,916 . 
Table 6b. HID Lamps - 400W Mercury Vapor Lamps and Replacements

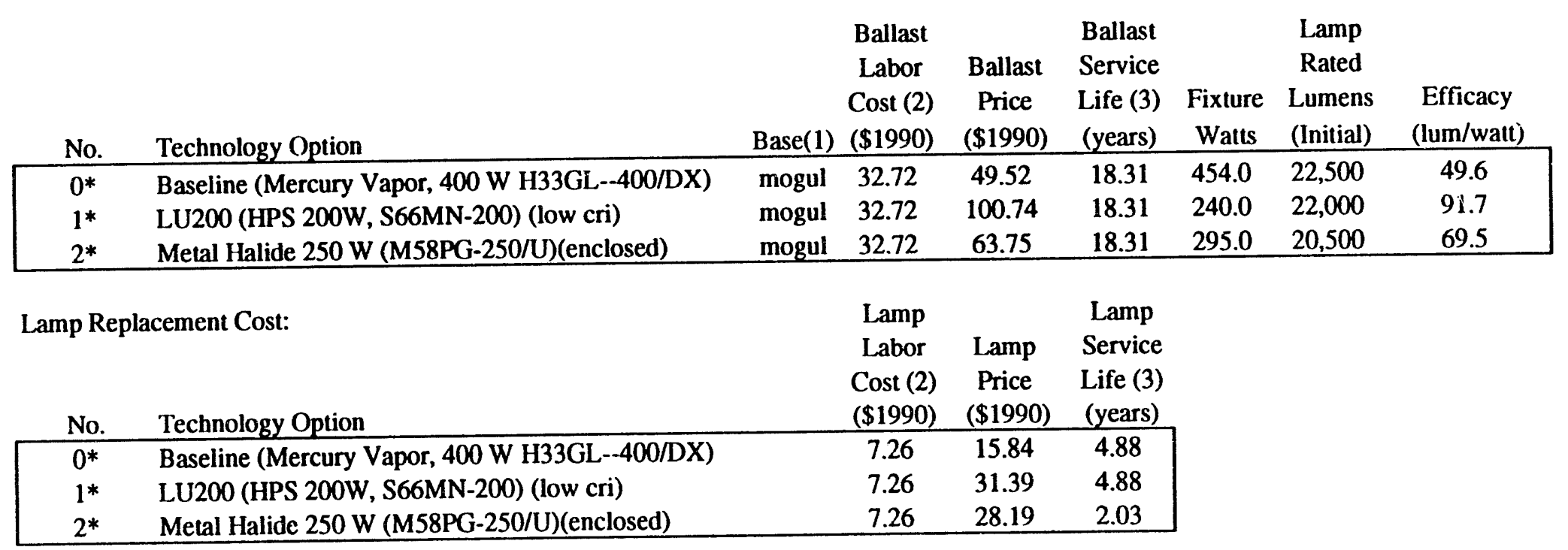

* Appears in Table 2.

(1) Mogul is a $40 \mathrm{~mm}(119 / 32 ")$ screw in base.

(2) Labor Rates ( $\$ 1990$ per hour) $=\$ 25.63$ (electrician helper), $\$ 36.69$ (electrician)--it is assumed that the time for

lamp replacement is charged at electrician helper rate, and the time for ballast replacement is evenly split between the electrician and helper.

(3) Calculated using annual lighting hours of $4,916$. 
Table 6c. HID Lamps - 1000W Mercury Vapor Lamps and Replacements

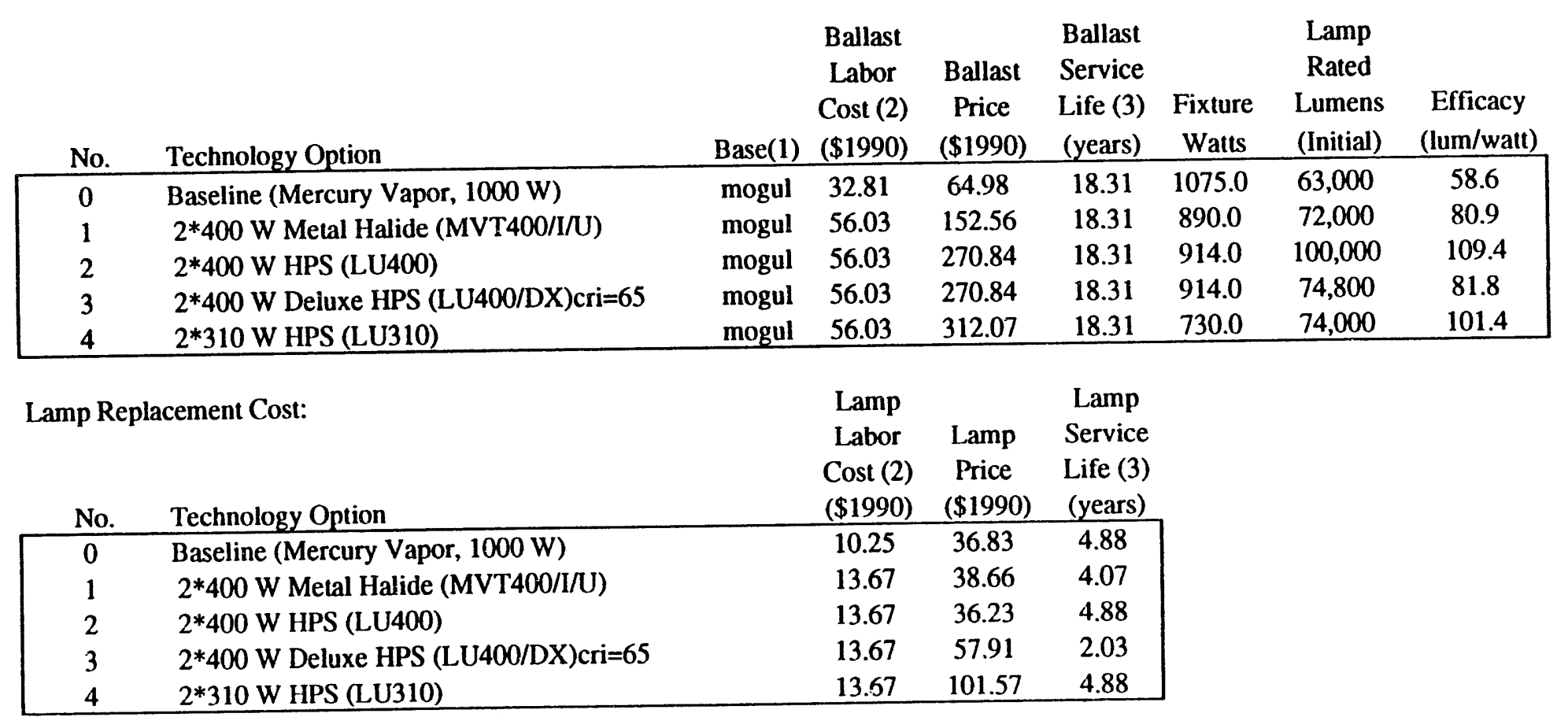

(1) Mogul is a $40 \mathrm{~mm}(119 / 32 ")$ screw in base.

(2) Labor Rates ( $\$ 1990$ per hour) $=\$ 25.63$ (electrician helper), $\$ 36.69$ (electrician)--it is assumed that the time for lamp replacement is charged at electrician helper rate, and the time for ballast replacement is evenly split between the electrician and helper.

(3) Calculated using annual lighting hours of 4,916 . 


\section{Saturation Data for Lighting Systems}

There are several ways to define technology shares: some of these are shares of connected load, shares of floorspace illuminated, shares of delivered lumens, and shares of supplied lumens (lumens out of lamps before fixture and room losses). COMMEND uses shares of delivered lumens. Within the COMMEND 4 framework, lighting systems (lamp/ballast combinations) are grouped into fixture types. Parallel to this type of representation, shares are defined in two levels: (1) the shares for the different fixture types, and (2) the shares for different systems within the fixture types.

\section{Fixture Shares of Lighting}

Previously, stock 1986 Energy Utilization Intensities (EUIs) were developed from the average of seven different utility studies[8]. The implied Lighting Power Densities (LPDs) were calculated using the annual lighting hours mentioned above. LPDs by fixture type were estimated by applying fixture shares of connected load developed from a large and detailed set of audit data collected in 1986 [9]. Table 7 gives LPDs for the building stock for the year 1986, and new buildings (sales) for 1986 and 1995. Using this information and efficacies from Tables 4, 5 and 6 for lamp/ballast combinations, data can be converted to the COMMEND format. The following equations show the derivation of fixture shares using information on connected load. An average efficacy for each fixture type has to be developed using the shares within fixture types presented in the following section. The average efficacy for a fixture type for a given building type is:

$$
\text { Average Efficacy } y_{i, b}=\frac{1}{\sum_{j=1}^{m} \text { System Share }_{j} * \frac{1}{\text { System Efficacy }_{j}{ }^{*} \text { Luminaire Efficiency }_{j}{ }^{*} \text { Room Factor }_{b}}}
$$

where,
$\mathrm{b}$ : index for building type
$i$ : index for fixture type
$\mathrm{j}$ : index for system type
$\mathrm{m}$ : number of system types within a fixture type. 
Illuminance delivered and fixture share for each fixture type and building type can be calculated as follows:

$$
\text { Illuminance Delivered }{ }_{i, b}=\text { Connected Load }_{i, b} * \text { Average Efficacy }_{i, b}
$$

$$
\text { Fixture Share }_{i, b}=\frac{\text { Illuminance Delivered }_{i, b}}{\sum_{i=1}^{n} \text { Illuminance Delivered }_{i, b}}
$$

where,

$\mathrm{b}$ : index for building type

$\mathrm{i}$ : index for fixture type

$\mathrm{n}:$ number of fixture types.

Stock and new fixture shares for 1986, and new fixture shares for 1995 are presented in Table 8. These shares on Table 8 are developed using data from Table 7 as discussed above. The 1995 data in Tables 7 and 8 are to be used as a calibration check for the equations determining future new-construction shares. Future trends are input to COMMEND as equations by the users. 
Table 7. Lighting Power Density by Building Type (W/sqft)(1)

1986 Stock

\begin{tabular}{|lcccc|}
\multicolumn{1}{l}{ fluo } & incand & hid & other \\
\hline small office & 1.32 & 0.17 & 0.04 & 0.00 \\
large office & 1.00 & 0.13 & 0.02 & 0.00 \\
restaurant & 0.58 & 0.54 & 0.00 & 0.01 \\
retail & 0.94 & 0.32 & 0.00 & 0.00 \\
grocery & 1.66 & 0.14 & 0.00 & 0.00 \\
warehouse & 0.58 & 0.12 & 0.10 & 0.00 \\
school & 0.61 & 0.13 & 0.01 & 0.00 \\
college & 1.44 & 0.12 & 0.00 & 0.00 \\
health & 0.64 & 0.11 & 0.00 & 0.00 \\
lodging & 0.07 & 0.36 & 0.00 & 0.00 \\
miscellaneous & 0.61 & 0.18 & 0.03 & 0.00 \\
\hline
\end{tabular}

1986 sales

\begin{tabular}{|lcccc|}
\hline & fluo & incand & hid & other \\
\hline small office & 1.32 & 0.17 & 0.03 & 0.00 \\
large office & 1.00 & 0.13 & 0.02 & 0.00 \\
restaurant & 0.58 & 0.54 & 0.00 & 0.01 \\
retail & 0.93 & 0.32 & 0.00 & 0.00 \\
grocery & 1.66 & 0.14 & 0.00 & 0.00 \\
warehouse & 0.58 & 0.12 & 0.08 & 0.00 \\
school & 0.61 & 0.13 & 0.01 & 0.00 \\
college & 1.44 & 0.12 & 0.00 & 0.00 \\
health & 0.64 & 0.11 & 0.00 & 0.00 \\
lodging & 0.07 & 0.36 & 0.00 & 0.00 \\
miscellaneous & 0.61 & 0.18 & 0.02 & 0.00 \\
\hline
\end{tabular}

\begin{tabular}{|lcccc|}
\multicolumn{1}{rc}{1995 sales } & fluo & incand & hid & other \\
\hline small office & 1.01 & 0.13 & 0.03 & 0.00 \\
large office & 0.77 & 0.10 & 0.01 & 0.00 \\
restaurant & 0.44 & 0.43 & 0.00 & 0.01 \\
retail & 0.72 & 0.26 & 0.00 & 0.00 \\
grocery & 1.27 & 0.12 & 0.00 & 0.00 \\
warehouse & 0.44 & 0.09 & 0.07 & 0.00 \\
school & 0.46 & 0.11 & 0.01 & 0.00 \\
college & 1.10 & 0.09 & 0.00 & 0.00 \\
health & 0.49 & 0.09 & 0.00 & 0.00 \\
lodging & 0.06 & 0.29 & 0.00 & 0.00 \\
miscellaneous & 0.47 & 0.14 & 0.02 & 0.00 \\
\hline
\end{tabular}

(1) The numbers apply to the total floor area for the building type, and not to the area which has the particular fixture type. These numbers were developed from [8] and [9]. 
Table 8. Fixture Shares of Delivered Lumens (1)

1986 Stock

\begin{tabular}{|lcccc|}
\multicolumn{1}{c}{ fluo } & incand & hid & other \\
\hline small office & $96 \%$ & $2 \%$ & $2 \%$ & $0 \%$ \\
large office & $96 \%$ & $2 \%$ & $1 \%$ & $0 \%$ \\
restaurant & $84 \%$ & $15 \%$ & $0 \%$ & $1 \%$ \\
retail & $94 \%$ & $6 \%$ & $0 \%$ & $0 \%$ \\
grocery & $98 \%$ & $2 \%$ & $0 \%$ & $0 \%$ \\
warehouse & $86 \%$ & $3 \%$ & $11 \%$ & $0 \%$ \\
school & $95 \%$ & $4 \%$ & $1 \%$ & $0 \%$ \\
college & $98 \%$ & $2 \%$ & $0 \%$ & $0 \%$ \\
health & $97 \%$ & $3 \%$ & $0 \%$ & $0 \%$ \\
lodging & $51 \%$ & $49 \%$ & $0 \%$ & $0 \%$ \\
miscellaneous & $92 \%$ & $5 \%$ & $3 \%$ & $0 \%$ \\
\hline
\end{tabular}

1986 sales

\begin{tabular}{|lcccc|}
\hline & fluo & incand & hid & other \\
\hline small office & $96 \%$ & $2 \%$ & $2 \%$ & $0 \%$ \\
large office & $97 \%$ & $2 \%$ & $1 \%$ & $0 \%$ \\
restaurant & $85 \%$ & $14 \%$ & $0 \%$ & $1 \%$ \\
retail & $94 \%$ & $6 \%$ & $0 \%$ & $0 \%$ \\
grocery & $98 \%$ & $2 \%$ & $0 \%$ & $0 \%$ \\
warehouse & $88 \%$ & $3 \%$ & $8 \%$ & $0 \%$ \\
school & $95 \%$ & $4 \%$ & $1 \%$ & $0 \%$ \\
college & $99 \%$ & $1 \%$ & $0 \%$ & $0 \%$ \\
health & $97 \%$ & $3 \%$ & $0 \%$ & $0 \%$ \\
lodging & $52 \%$ & $48 \%$ & $0 \%$ & $0 \%$ \\
miscellaneous & $93 \%$ & $5 \%$ & $3 \%$ & $0 \%$ \\
\hline
\end{tabular}

\begin{tabular}{|lcccc|}
\multicolumn{1}{c}{1995 sales } & \multicolumn{1}{c}{ fluo } & incand & hid & other \\
\hline small office & $96 \%$ & $3 \%$ & $2 \%$ & $0 \%$ \\
large office & $96 \%$ & $3 \%$ & $1 \%$ & $0 \%$ \\
restaurant & $82 \%$ & $16 \%$ & $0 \%$ & $1 \%$ \\
retail & $93 \%$ & $7 \%$ & $0 \%$ & $0 \%$ \\
grocery & $98 \%$ & $2 \%$ & $0 \%$ & $0 \%$ \\
warehouse & $88 \%$ & $4 \%$ & $9 \%$ & $0 \%$ \\
school & $95 \%$ & $5 \%$ & $1 \%$ & $0 \%$ \\
college & $98 \%$ & $2 \%$ & $0 \%$ & $0 \%$ \\
health & $96 \%$ & $4 \%$ & $0 \%$ & $0 \%$ \\
lodging & $50 \%$ & $50 \%$ & $0 \%$ & $0 \%$ \\
miscellaneous & $92 \%$ & $6 \%$ & $3 \%$ & $0 \%$ \\
\hline
\end{tabular}

(1) Shares represent \%of delivered lumens by fixture type. Shares may not add to $100 \%$ due to rounding error. 


\section{System Shares within Fixture Types}

LBL has developed system shares of illuminance delivered within a fixture type. This is done using a combination of survey data collected by the Lighting Research Institute [10], DOE [5], and the Bureau of the Census [11]. Table 9 gives fluorescent shares for the building stock in 1986, Table 10 gives shares for new buildings (sales) in 1986, and Table 11 gives the shares for new buildings (sales) in 1995. Table 12 gives standard fixture system shares of illuminance delivered in 1986 and 1995. It should be noted that the the shares for 4-foot and 8-foot fixtures add up to approximately 100 . If the intention is to treat 4-foot and 8-foot fixtures separately, then shares within each category can be developed using Table 9.

Shares for energy-efficient magnetic ballast were estimated based on work by Geller and Miller [12]. Market shares for 1987 sales were adjusted by Geller and Miller to represent market shares if state standards did not exist in 1987. By the end of 1987, standards prohibiting sale of inefficient core-coil ballasts existed in five states representing about one quarter of the U.S. population (California, New York, Massachusetts, Connecticut, and Florida). Without the standards, market share for energy efficient magnetic ballasts were estimated to be about 10 percent. Considering the fact that energy efficient magnetic ballast have been in the market since the seventies, the stock saturation in this report for this ballast type was estimated to be about 10 percent. The market share in sales is estimated to be 33 percent which is 25 percent (for states with standards), plus 8 percent (for states with no standards for ballasts). Estimates based on NBECS [5] give higher shares for energy efficient magnetic ballasts. This may be because, when a survey response indicates that this type of ballast is used in the building, it is sometimes interpreted to mean that the whole building is utilizing such ballasts.

The 1995 data in Table 11 can be used as a calibration check for the equations determining future shares. In COMMEND either trend equations or discrete choice equations can be used to determine future shares. 
Table 9. Fluorescent System Shares--1986 Stock

\begin{tabular}{|c|c|c|c|}
\hline System & $\begin{array}{l}\text { Relative * } \\
\text { Share (\%) }\end{array}$ & $\begin{array}{c}\text { Market } \\
\text { Share (\%) }\end{array}$ & Notes \\
\hline 4-Foot Lamps & 78.3 & & Census Data \\
\hline Std Ballast & 90 & & (100-EE Magnetic-Electronic-Cathode Cut.)\% \\
\hline Std F40 & 86.3 & 60.8 & Census Data \\
\hline ES 34W & 13.7 & 9.7 & $(100-$ Std F40 - T-8)\% \\
\hline EE Mag Ballast & 9.7 & & Estimated based on [12] \\
\hline Std F40 & 86.2 & 6.5 & \\
\hline ES 34W & 13.7 & 1.0 & \\
\hline $\mathrm{T}-8$ & 0.1 & 0.0 & \\
\hline Cath Cut Ballast & 0 & & LBL Estimate \\
\hline Std F40 & 86.2 & 0 & \\
\hline ES 34W & 13.7 & 0 & \\
\hline $\mathrm{T}-8$ & 0.1 & 0 & \\
\hline Elect Ballast & 0.3 & & Census Data \\
\hline Std F40 & 86.2 & 0.2 & \\
\hline ES $34 \mathrm{~W}$ & 13.7 & 0 & \\
\hline $\mathrm{T}-8$ & 0.1 & 0 & \\
\hline 8-Foot Lamps & 12.9 & & Census Data \\
\hline Std Ballast & 90 & & (100 - EE Magnetic - Electronic)\% \\
\hline Std F96 & 55 & 6.4 & NBECS 1986 \\
\hline ES F96 & 45 & 5.2 & NBECS 1986 \\
\hline EE Mag Ballast & 9.7 & & Estimated based on [12] \\
\hline Std F96 & 55 & 0.7 & \\
\hline ES F96 & 45 & 0.6 & \\
\hline Elect Ballast & 0.3 & & Census Data \\
\hline Std F96 & 55 & 0 & \\
\hline ES F96 & 45 & 0 & \\
\hline 8-foot High Out. & 7.4 & & Census Data \\
\hline Std Ballast & 90 & & (100 - EE Magnetic - Electronic)\% \\
\hline Std F96 & 55 & 3.7 & NBECS 1986 \\
\hline ES F96 & 45 & 3.0 & NBECS 1986 \\
\hline EE Mag Ballast & 9.7 & & Estimated based on [12] \\
\hline Std F96 & 55 & 0.4 & \\
\hline ES F96 & 45 & 0.3 & \\
\hline Elect Ballast & 0.3 & & Census Data \\
\hline Std F96 & 55 & 0 & \\
\hline ES Fy6 & 45 & 0 & \\
\hline
\end{tabular}

* Relative Share stands for share within fixture type, ballast type or lamp type 
Table 10. Fluorescent System Shares--1986 New-Construction

\begin{tabular}{|c|c|c|c|}
\hline System & $\begin{array}{c}\text { Relative* } \\
\text { Share (\%) }\end{array}$ & $\begin{array}{c}\text { Market } \\
\text { Share }(\%)\end{array}$ & Notes \\
\hline 4-Foot Lamps & 78.3 & & Census Data \\
\hline Std Ballast & 66.2 & & (100-EE Magnetic-Electronic-Cathode Cut.)\% \\
\hline Std F40 & 86.4 & 44.8 & Census Data \\
\hline ES 34W & 13.6 & 7.0 & $(100-$ Std F40 - T-8)\% \\
\hline EE Mag Ballast & 33.0 & & Estimated based on [12] \\
\hline Std F40 & 86.2 & 22.3 & \\
\hline ES $34 \mathrm{~W}$ & 13.6 & 3.5 & \\
\hline T.8 & 0.2 & 0.1 & \\
\hline Cath Cut Ballast & 0 & & Estimate \\
\hline Std F40 & 86.2 & 0 & \\
\hline ES 34W & 13.6 & 0 & \\
\hline $\mathrm{T} \cdot 8$ & 0.2 & 0 & \\
\hline Elect Ballast & 0.8 & & Census Data \\
\hline Std F40 & 86.2 & 0.5 & \\
\hline ES 34W & 13.6 & 0.1 & \\
\hline T-8 & 0.2 & 0 & \\
\hline 8-Foot Lamps & 13.0 & & Census Data \\
\hline Std Ballast & 66.2 & & (100 - EE Magnetic - Electronic)\% \\
\hline Std F96 & 55 & 4.7 & NBECS 1986 \\
\hline ES F96 & 45 & 3.9 & NBECS 1986 \\
\hline EE Mag Ballast & 33.0 & & Estimated based on [12] \\
\hline Std F96 & 55 & 2.4 & \\
\hline ES F96 & 45 & 1.9 & \\
\hline Elect Ballast & 0.8 & & Census Data \\
\hline Std F96 & 55 & 0.1 & \\
\hline ES F96 & 45 & 0.0 & \\
\hline 8-foot High Out. & 7.5 & & Census Data \\
\hline Std Ballast & 66.2 & & (100 - EE Magnetic - Electronic)\% \\
\hline Std F96 & 55 & 2.7 & NBECS 1986 \\
\hline ES F96 & 45 & 2.2 & NBECS 1986 \\
\hline EE Mag Ballast & 33.0 & & Estimated based on [12] \\
\hline Std F96 & 55 & 1.4 & \\
\hline ES F96 & 45 & 1.1 & \\
\hline Elect Ballast & 0.8 & & Census Data \\
\hline Std F96 & 55 & 0 & \\
\hline ES F96 & 45 & 0 & \\
\hline
\end{tabular}

* Relative Share stands for share within fixture type, ballast type or lamp type 
Table 11. Fluorescent System Shares--1995 New-Construction

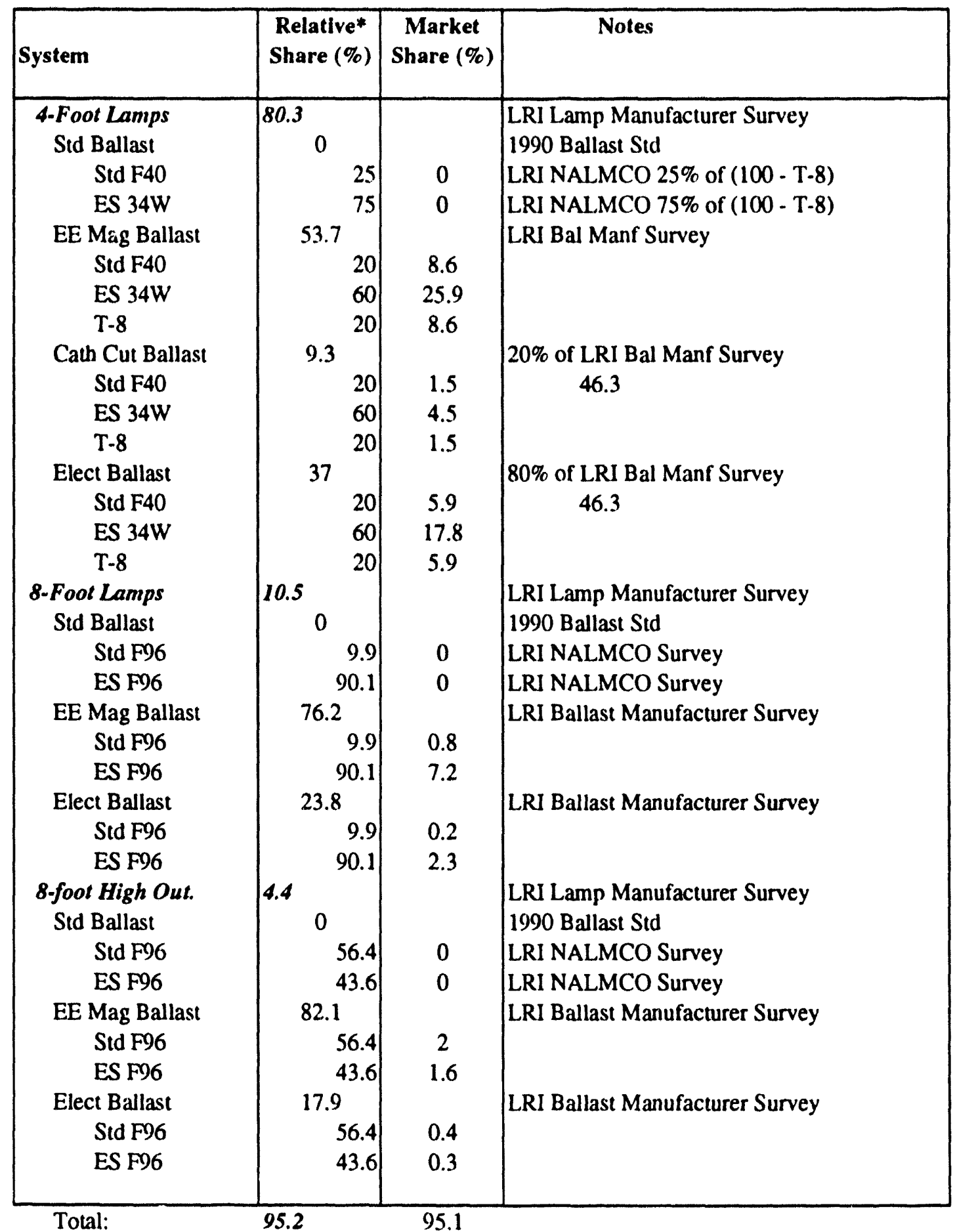

* Relative Share stands for share within fixture type, ballast type or lamp type 
Table 12. Standard Fixture Shares--Stock/New-Construction

\begin{tabular}{|c|c|c|c|}
\hline System & $\begin{array}{c}\text { Relative } \\
\text { Share }(\%)\end{array}$ & $\begin{array}{c}\text { Market } \\
\text { Share (\%) }\end{array}$ & Notes \\
\hline Incandescent & 100 & & \\
\hline General Service & 80 & & Census Data \\
\hline$>150$ & 2 & 1.6 & Census Data \\
\hline $15-150$ Std & 93 & 74.4 & $(100-$ others $) \%$ \\
\hline Reduced Wattage & 5 & 4 & Estimate \\
\hline Halogen & 0 & 0 & \\
\hline Reflector & 10 & & Census Data \\
\hline Standard Par & 33.2 & 3.3 & $(100$-others $) *$ census $=42 /(42+53)$ \\
\hline Standard R & 41.8 & 4.2 & $(100$-others $) *$ census $=53 /(42+53)$ \\
\hline Reduced Wattage Par/R & 20 & 2 & Estimate \\
\hline Halogen & 5 & 0.5 & census \\
\hline Halogen Infrared (IR) & 0 & 0 & \\
\hline Other & 10 & & Census Data \\
\hline Std & 100 & 10 & \\
\hline Compact Fluor. & 0 & 0 & \\
\hline
\end{tabular}

1995

\begin{tabular}{|c|c|c|c|}
\hline System & $\begin{array}{c}\text { Relative* } \\
\text { Share (\%) }\end{array}$ & $\begin{array}{c}\text { Market } \\
\text { Share (\%) }\end{array}$ & \\
\hline Incandescent & 80 & & \\
\hline General Service & 80 & & Census Data (1989) \\
\hline$>150$ & 2 & 1.3 & Census Data \\
\hline $15-150$ Std & 43 & 27.5 & 100 - others \\
\hline Reduced Wattage & 50 & 32.0 & Estimate \\
\hline Halogen & 5 & 3.2 & Estimate \\
\hline Reflector & 10 & & Census Data (1989) \\
\hline Standard Par & 13.3 & 1.1 & $(100 \text {-others })^{*}$ census $=42 /(42+53)$ \\
\hline Standard R & 16.7 & 1.3 & $(100 \text {-others })^{*}$ census $=53 /(42+53)$ \\
\hline Reduced Wattage Par $/ \mathbb{R}$ & 30 & 2.4 & Estimate \\
\hline Halogen & 30 & 2.4 & Estimate \\
\hline Halogen Infrared (IR) & 10 & 0.8 & Estimate \\
\hline Other & 10 & & Census Data (1989) \\
\hline Std & 100 & 8.0 & \\
\hline Compact Fluor. & 20 & 20.0 & LRI Lamp Manf Survey \\
\hline
\end{tabular}

* Relative Share stands for share within fixture type or lamp type 


\section{LUMINAIRE DATA}

Luminaire efficiency stands for the percentage of the light output from the lamps that is actually emitted from the fixture. In other words, it models losses due to the luminaire. These losses are affected by the fixture geometry and also the reflectances of the interior surfaces of the luminaire. Table 13 presents efficiencies for the important fixture types. Table 14a characterizes the fixture distribution over the market in the year 1986. Table $14 \mathrm{~b}$ presents an estimate of future trends.

The luminaire market is expected to move from lensed troffers to recessed parabolic luminaires as more office spaces have VDT screens. Less 4-lamp luminaires will be used as the lamp/ballast efficacy increases. 
Table 13. Luminaire Efriciencies

\begin{tabular}{|l|cccc|}
\hline \multicolumn{1}{l|}{ Fluorescent } & 1-lamp & 2-lamp & 3-lamp & 4-lamp \\
Lensed Troffer & $54 \%$ & $59 \%$ & $64 \%$ & $62 \%$ \\
Wraparound & & & & \\
& $80 \%$ & $72 \%$ & $70 \%$ & $62 \%$ \\
Parabolic & & & & \\
$\quad$ Narrow\&Medium & & & $63 \%$ & $58 \%$ \\
$\quad$ Wide & $61 \%$ & $61 \%$ & $64 \%$ & \\
\hline Standard Base & $72 \%$ & $71 \%$ & & \\
Incandescent & & & & \\
$\quad$ General Service & & & & \\
$\quad$ Reflector & $50 \%$ & & & \\
Compact Fluorescent & $50 \%$ & & & \\
\hline HID & $48 \%$ & $57 \%$ & & \\
$\quad$ Round-No Refractor & & & & \\
$\quad$ Round-Refractor & $68 \%$ & & & \\
$\quad$ Square & $77 \%$ & & & \\
Indirect & $60 \%$ & & & \\
\hline
\end{tabular}

Source: LBL Luminaire Database and New York State Energy Office Luminaire Database 
Table 14a. Fluorescent Fixture Saturation as a Function of of Number of Lamps per Fixture (1986)

\begin{tabular}{|c|c|c|c|c|c|}
\hline Type & All & 1-lamp & 2-lamp & 3-lamp & 4-lamp \\
\hline Lensed Troffer & $42.0 \%$ & $0.4 \%$ & $7.6 \%$ & $11.8 \%$ & $22.3 \%$ \\
\hline Wraparound & $17.0 \%$ & $0.9 \%$ & $11.4 \%$ & $0.2 \%$ & $4.6 \%$ \\
\hline Parabolic & $18.0 \%$ & $0.9 \%$ & $5.4 \%$ & $7.2 \%$ & $4.5 \%$ \\
\hline Other & $23.0 \%$ & $\mathrm{n} / \mathrm{a}$ & $\mathrm{n} / \mathrm{a}$ & n/a & n/a \\
\hline
\end{tabular}

Source: Bureau of Census [11]

Table 14b. Fluorescent Fixture Saturation as a Function of of Number of Lamps per Fixture (1995)(1)

Type
\begin{tabular}{|l|c|cccc|}
\hline Lensed Troffer & $22.0 \%$ & $\mathrm{n} / \mathrm{a}$ & $\mathrm{n} / \mathrm{a}$ & $\mathrm{n} / \mathrm{a}$ & 4-lamp \\
Wraparound & $23.0 \%$ & $\mathrm{n} / \mathrm{a}$ & $\mathrm{n} / \mathrm{a}$ & $\mathrm{n} / \mathrm{a}$ & $\mathrm{n} / \mathrm{a}$ \\
Parabolic & $32.0 \%$ & $1.6 \%$ & $12.8 \%$ & $14.4 \%$ & $3.2 \%$ \\
Other & $23.0 \%$ & $\mathrm{n} / \mathrm{a}$ & $\mathrm{n} / \mathrm{a}$ & $\mathrm{w} / \mathrm{a}$ & $\mathrm{n} / \mathrm{a}$ \\
\hline
\end{tabular}

n/a : not available

(1) LBL estimates 


\section{CONTROLS DATA}

Controls generally change the use pattern of the connected load, and decrease consumption by customizing operating hours to user needs. Controls range from simple mechanical timeclocks to sophisticated multi-level electronic devices that interface with a building's energy management system. The types of controls covered here are: programmable timers, lumen maintenance/dimming, occupancy sensors and daylighting/dimming.

Programmable timers provide time-based control of lighting equipment. The usual method of implementation is a system of low-voltage relays controlled by a programmable timeclock. To accommodate off-hours lighting needs, systems usually have overrides, so lights can be turned on by building occupants either by low-voltage switches or telephone procedures. In this report, timers are assumed to be multi-level and are applied to both fluorescent and incandescent lights.

Lumen maintenance controls limit power and light output when fluorescent lamps are new and the fixtures are clean. Without controls, light output decreases as the lamps age and dirt accumulates on the fixture reflector and/or lens. With lumen maintenance dimming controls, power is gradually increased over time until full power operation occurs when it is time to replace the lamps and clean the fixtures. This light output remains fairly constant throughout the lamp lifetime. This option is applied to full-size fluorescent lamps only (not compact fluorescent lamps).

Occupancy sensors are activated by the presenc: or absence of people in the field of view. The lights in the controlled zone are turned on automatically when a person enters the area, and are turned off after the room is unoccupied for a set period of time. There are two basic types of sensors: passive infrared and ultrasonic. In this report, this control option applies to fluorescent and incandescent lights.

Daylighting controls use a photocell with a dimming system to provide a fixed light level at the work plane by decreasing the amount of light as daylight levels increase and increasing it with reduced daylight. This option is applied to full-size fluorescent lamps only. Daylighting affects both operating hours and lighting load.

Other types of controls, such as two-level switching, incandescent or compact fluorescent dimmers, and stepped switching are not covered in this report. 
Effectiveness, saturations, and penetration trends of control options for new buildings and retrofit situations by building type are supplied as inputs to COMMEND. Table 15 presents savings, price, and applicability of controls. For timers and occupancy sensors, percentage savings, applicable percent floor area, and applicable building types are determined from manufacturer estimates and staff experience. ${ }^{5}$ Energy savings from lumen maintenance accrue in watts/sqft during the early part of a lamp's lifetime. In California's Title 24 energy code, controls credits allow 0.1 watts/sqft savings for lumen maintenance. Energy savings from daylighting accrue in reduced lighting hours for onoff controls and in watts/sqft for dimming. This is translated into percentage savings, estimated by the LBL Lighting Systems Research Group. Daylighting controls are applied to one-half of the building perimeter floor area taken from NBECS 1986.

\footnotetext{
5 Timers: Dave Peterson, GE Wiring Devices, Rhode Island and LBL's Lighting Systems Research Group. Occupancy sensors: Jerry Mix, The Watt-Stopper, Santa Clara, California, and LBL's Lighting Systems Research Group.
} 
Table 15. Impacts, Costs and Applicability of Controls

\section{TIMERS}

\begin{tabular}{|c|c|c|c|c|}
\hline & $\begin{array}{l}\text { Savings } \\
\text { Fraction }\end{array}$ & $\begin{array}{l}\text { Applicable } \\
\text { Fraction(1) }\end{array}$ & $\begin{array}{c}\text { Applicable } \\
\text { Technology (2) }\end{array}$ & $\begin{array}{l}\text { Price(3) Notes } \\
\text { (\$/sqft) }\end{array}$ \\
\hline Small Off & 0.23 & 1 & $F+I$ & 0.30 \\
\hline Lg Off & 0.23 & 1 & $F+I$ & 0.30 \\
\hline Rest & 0.00 & 1 & $F+I$ & 0.00 \\
\hline Retail & 0.10 & 1 & $F+I$ & 0.25 \\
\hline Grocery & 0.10 & 1 & $F+I$ & 0.25 \\
\hline Warehse & 0.30 & 1 & $F+I$ & 0.25 \\
\hline School & 0.15 & 1 & $F+I$ & 0.45 \\
\hline College & 0.15 & 1 & $F+I$ & 0.45 \\
\hline Health & 0.00 & 1 & $F+I$ & 0.00 \\
\hline Lodging & 0.00 & 1 & $F+I$ & 0.00 \\
\hline Misc & 0.15 & 1 & $\mathrm{~F}+\mathrm{I}$ & 0.30 \\
\hline
\end{tabular}

\section{TIMERS + LUMEN MAINTENANCE}

Savings Applicable Applicable Price(3) Notes Fraction Fraction(1) Technology(2) (\$/sqf)

\begin{tabular}{|llllll|}
\hline Small Off & 0.33 & 1 & $\mathrm{~F}$ & 0.57 & Assumes 10\% savings; cost= \\
Lg Off & 0.33 & 1 & $\mathrm{~F}$ & 0.49 & 30c/sq ft where F LPD=1.0 \\
Rest & 0.10 & 1 & $\mathrm{~F}$ & 0.16 & No Timer \\
Retail & 0.20 & 1 & $\mathrm{~F}$ & 0.39 & \\
Grocery & 0.20 & 1 & $\mathrm{~F}$ & 0.59 & \\
Warehse & 0.40 & 1 & $\mathrm{~F}$ & 0.31 & \\
School & 0.25 & 1 & $\mathrm{~F}$ & 0.52 & \\
College & 0.25 & 1 & $\mathrm{~F}$ & 0.70 & \\
Health & 0.10 & 1 & $\mathrm{~F}$ & 0.16 & No Timer \\
Lodging & 0.10 & 1 & $\mathrm{~F}$ & 0.02 & No Timer \\
Misc & 0.25 & 1 & $\mathrm{~F}$ & 0.38 & \\
\hline
\end{tabular}

(1) Fraction of floorspace to which the technology option is applicable.

(2) $\mathrm{F}=$ fluorescent, and I = incandescent.

(3) Capital cost of the technology option per applicable square foot. 
Table 15. Impacts, Costs and Applicability of Controls (continued)

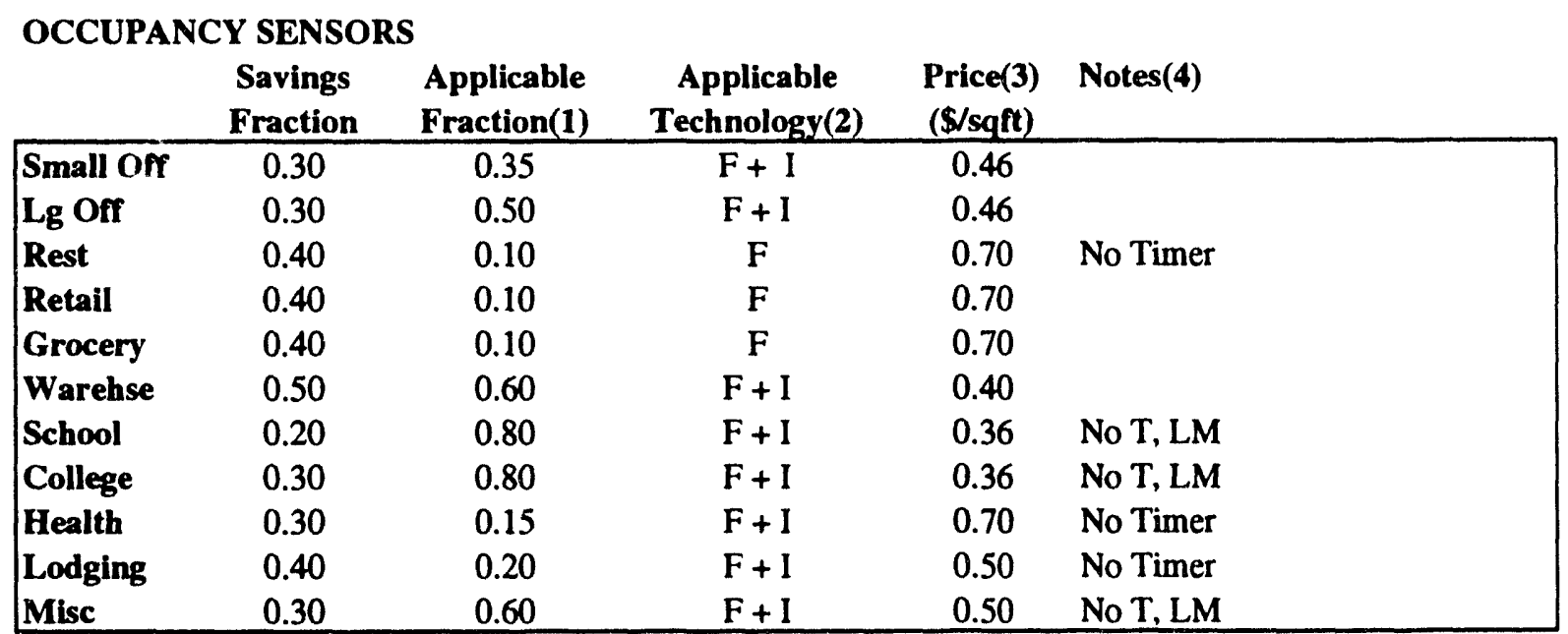

DAYLIGHTING

\begin{tabular}{|lccccl|}
\hline Savings & $\begin{array}{c}\text { Applicable } \\
\text { Fraction }\end{array}$ & $\begin{array}{c}\text { Applicable } \\
\text { Fraction(1) }\end{array}$ & $\begin{array}{c}\text { Price(3) } \\
\text { Technology(2) }\end{array}$ & $\begin{array}{c}\text { Notes(4) } \\
\text { (\$/sqft) }\end{array}$ & \\
\hline Lg Off & 0.35 & 0.28 & F & 1.10 & \\
Rest & 0.35 & 0.28 & F & 1.10 & \\
Retail & 0.35 & 0.00 & F & 1.10 & No T,OS \\
Grocery & 0.35 & 0.00 & F & 1.10 & \\
Warehse & 0.35 & 0.00 & F & 1.10 & \\
School & 0.35 & 0.00 & F & 1.10 & \\
College & 0.35 & 0.20 & F & 1.10 & No T, LM, OS \\
Health & 0.35 & 0.20 & F & 1.10 & No T, LM \\
Lodging & 0.35 & 0.25 & F & 1.10 & No Timer \\
Misc & 0.35 & 0.00 & F & 1.10 & No Timer \\
\hline
\end{tabular}

(1) Fraction of floorspace to which the technology option is applicable.

(2) $\mathrm{F}=$ fluorescent and $\mathrm{I}=$ incandescent.

(3) Capital cost of the technology option per applicable square foot.

(4) $\mathrm{T}=$ timers, $\mathrm{LM}=$ lumen maintenance, $\mathrm{OS}=$ occupancy sensor 


\section{LIGHTING/HVAC INTERACTIONS}

The secondary effect of lighting energy reduction on building energy use is due to lighting/HVAC interactions. This report looks at changes in energy use for cooling and heating but does not address the additional benefits due to reduced equipment sizing. The general belief within expert groups advocating energy-efficiency is that there are additional net benefits from lighting reduction because of reduced cooling demand. LBL research shows that there are net benefits for cooling dominated regions and net penalties for heating dominated regions.

For ten building types in ten climate zones, prototype buildings are simulated using the DOE-2 building energy analysis program. Lighting/HVAC coincidence factors for each building/climate combination are developed. Cooling and heating coincidence factors are defined as the annual fractions of lighting energy saved resulting in reduced cooling and increased heating loads respectively.

\section{Coincidence Factors for Prototypical Buildings}

The attached tables and figures show DOE-2 simulation results for Heating and Cooling Coincidence Factors (HCF and CCF) for varying lighting power densities (W/ $\left./ \mathrm{ft}^{2}\right)$ for ten commercial building types (large and medium offices, large retail, large and small hotels, hospital, fast-food and sit-down restaurants, supermarket and secondary school) in ten cities (Charleston, Chicago, Lake Charles, Miami, Minneapolis, New York, Pasadena, Phoenix, San Francisco, and Washington ).

The Heating and Cooling Coinciuence Factors can be conceptualized as the coincidence of a building's lighting load and its space conditioning load. In reality, since the load due to heat gain from lights may be delayed for hours, these "coincidence factors" are actually calculated using DOE-2 hour-by-hour simulations where the lighting power density of the building is varied and the resultant changes in annual heating and cooling loads noted.

The prototypical building used for this study are a subset of the 481 prototypical commercial buildings described for the GRI Cogeneration Study [13]. The ten selected cities represent major climate variations within the U.S. Depending on the building type and vintage, the ten prototypes vary by city in size, shell characteristics, and internal conditions. The Cogeneration Study data base did not include five of the cities. Those cities were modeled with the appropriate weather tape, but using prototypes defined for the nearest available location : Chicago for Minneapolis, Miami for Charleston, Philadelphia for Washington, Houston for Lake Charles, and Los Angeles for Pasadena.

Both building vintages defined in the Cogeneration study were simulated - (1) Current, representing post-1980s construction following the ASHRAE-90.75 building energy 
standard, and (2) Old, representing the average characteristics of all buildings built prior to 1980. For each of the two restaurant prototypes (sit-down and fast-food), a single Average vintage was simulated. Since the coincidence factors must be expressed in terms of building loads, not HVAC-system or -plant loads, system variations were not studied. Building loads incorporating only the thermostat settings and the minimum fresh-air requirements were developed.

For each building-type/location/vintage combination, two simulations were done using the recently released DOE-2.1E program: (1) Average lighting power density, and (2) Low lighting power density ( 0.667 times the Average). The results of these simulations are shown in Tables 16a-j. Each table shows the changes in heating, cooling, and lighting loads from the base case. The last two columns show the heating (HCF) and cooling (CCF) coincidence factors calculated by dividing the change in space conditioning load by the change in lighting load.

The results are also plotted as bar charts in Figure 1. To illustrate the reverse relationship between HCF and CCF, the former are plotted as negative (e.g., increases the heating load) while the latter are plotted as positive (e.g., decreases the cooling load). Note that in the larger building types (large office, large retail, hospital, etc.) the sums of the HCF and CCF are constant across the ten locations, indicating that their space conditioning requirements are determined mostly by their internal loads rather than the climate or building shell. On the other hand, the smaller buildings, notably the small hotels, have coincidence factors that vary by location and vintage.

The plots also indicate that the coincidence factors of the larger buildings are more sensitive to the internal load than to differences in vintages. However, the coincidence factors for the smaller buildings vary more by vintage than by lighting power density. 
Table 16a. Heating and Cooling Coincidence Factors for Fast Foods Restaurant

\begin{tabular}{|ll|ccc|cc|}
\hline Location & Vintage & $\begin{array}{c}\Delta H L \\
(\mathrm{kBtu} / \mathrm{sf})\end{array}$ & $\begin{array}{c}\Delta \mathrm{CL} \\
(\mathrm{kBtw} / \mathrm{sf})\end{array}$ & $\begin{array}{c}\Delta \mathrm{LL} \\
(\mathrm{kBtw} / \mathrm{sf})\end{array}$ & HCF & CCF \\
\hline Charleston & Average & -1.14 & -14.12 & -16.26 & 0.070 & 0.868 \\
Chicago & Average & -2.62 & -12.78 & -16.26 & 0.161 & 0.786 \\
Lake Charles & Average & -0.81 & -14.46 & -16.26 & 0.050 & 0.889 \\
Miami & Average & -0.04 & -15.38 & -16.26 & 0.002 & 0.946 \\
Minneapolis & Average & -3.49 & -11.88 & -16.26 & 0.215 & 0.731 \\
New York & Average & -2.68 & -12.61 & -16.26 & 0.165 & 0.776 \\
Pasadena & Average & -0.66 & -14.59 & -16.26 & 0.041 & 0.897 \\
Phoenix & Average & -0.53 & -14.83 & -16.26 & 0.033 & 0.912 \\
San Francisco & Average & -1.47 & -13.62 & -16.26 & 0.090 & 0.838 \\
Washington & Average & -2.12 & -13.20 & -16.26 & 0.130 & 0.812 \\
\hline
\end{tabular}

Table 16b. Heating and Cooling Coincidence Factors for Hospital

\begin{tabular}{|c|c|c|c|c|c|c|}
\hline Location & Vintage & $\begin{array}{c}\Delta H L \\
(k B t u / s f)\end{array}$ & $\begin{array}{c}\triangle \mathrm{CL} \\
(\mathrm{kBtw} / \mathrm{sf})\end{array}$ & $\begin{array}{c}\Delta \mathrm{LL} \\
(\mathrm{kBtw} / \mathrm{sf})\end{array}$ & $\mathrm{HCF}$ & CCF \\
\hline Charleston & $\begin{array}{l}\text { Current } \\
\text { Old }\end{array}$ & $\begin{array}{l}-0.60 \\
-0.64\end{array}$ & $\begin{array}{l}-13.13 \\
-13.08\end{array}$ & $\begin{array}{l}-13.86 \\
-13.86\end{array}$ & $\begin{array}{l}0.043 \\
0.046\end{array}$ & $\begin{array}{l}0.947 \\
0.944\end{array}$ \\
\hline Chicago & $\begin{array}{l}\text { Current } \\
\text { Old }\end{array}$ & $\begin{array}{l}-0.78 \\
-1.51\end{array}$ & $\begin{array}{l}-12.96 \\
-12.16\end{array}$ & $\begin{array}{l}-13.86 \\
-13.86\end{array}$ & $\begin{array}{l}0.056 \\
0.109\end{array}$ & $\begin{array}{l}0.935 \\
0.877\end{array}$ \\
\hline Lake Charles & $\begin{array}{l}\text { Current } \\
\text { Old }\end{array}$ & $\begin{array}{l}-0.39 \\
-0.47\end{array}$ & $\begin{array}{l}-13.37 \\
-13.26\end{array}$ & $\begin{array}{l}-13.86 \\
-13.86\end{array}$ & $\begin{array}{l}0.028 \\
0.034\end{array}$ & $\begin{array}{l}0.965 \\
0.957\end{array}$ \\
\hline Miami & $\begin{array}{l}\text { Current } \\
\text { Old }\end{array}$ & $\begin{array}{l}-0.04 \\
-0.04\end{array}$ & $\begin{array}{l}-13.79 \\
-13.80\end{array}$ & $\begin{array}{l}-13.86 \\
-13.86\end{array}$ & $\begin{array}{l}0.003 \\
0.003\end{array}$ & $\begin{array}{l}0.995 \\
0.996\end{array}$ \\
\hline Minneapolis & $\begin{array}{l}\text { Current } \\
\text { Old }\end{array}$ & $\begin{array}{l}-0.96 \\
-1.87\end{array}$ & $\begin{array}{l}-12.78 \\
-11.79\end{array}$ & $\begin{array}{l}-13.86 \\
-13.86\end{array}$ & $\begin{array}{l}0.069 \\
0.135\end{array}$ & $\begin{array}{l}0.922 \\
0.851\end{array}$ \\
\hline New York & $\begin{array}{l}\text { Current } \\
\text { Old }\end{array}$ & $\begin{array}{l}-0.81 \\
-1.58\end{array}$ & $\begin{array}{l}-12.77 \\
-11.97\end{array}$ & $\begin{array}{l}-13.87 \\
-13.87\end{array}$ & $\begin{array}{l}0.058 \\
0.114\end{array}$ & $\begin{array}{l}0.921 \\
0.863\end{array}$ \\
\hline Pasadena & $\begin{array}{l}\text { Current } \\
\text { Old }\end{array}$ & $\begin{array}{l}-0.11 \\
-0.15\end{array}$ & $\begin{array}{l}-13.72 \\
-13.68\end{array}$ & $\begin{array}{l}-13.86 \\
-13.86\end{array}$ & $\begin{array}{l}0.008 \\
0.011\end{array}$ & $\begin{array}{l}0.990 \\
0.987\end{array}$ \\
\hline Phoenix & $\begin{array}{l}\text { Current } \\
\text { Old }\end{array}$ & $\begin{array}{l}-0.14 \\
-0.17\end{array}$ & $\begin{array}{l}-13.71 \\
-13.66\end{array}$ & $\begin{array}{l}-13.86 \\
-13.86\end{array}$ & $\begin{array}{l}0.010 \\
0.012\end{array}$ & $\begin{array}{l}0.989 \\
0.986\end{array}$ \\
\hline San Francisco & $\begin{array}{l}\text { Current } \\
\text { Old }\end{array}$ & $\begin{array}{l}-0.39 \\
-0.55\end{array}$ & $\begin{array}{l}-13.35 \\
-13.14\end{array}$ & $\begin{array}{l}-13.86 \\
-13.86\end{array}$ & $\begin{array}{l}0.028 \\
0.040\end{array}$ & $\begin{array}{l}0.963 \\
0.948\end{array}$ \\
\hline $\begin{array}{l}\text { Washington } \\
\text { " }\end{array}$ & $\begin{array}{l}\text { Current } \\
\text { Old }\end{array}$ & $\begin{array}{l}-0.47 \\
-1.02\end{array}$ & $\begin{array}{l}-13.29 \\
-12.68\end{array}$ & $\begin{array}{l}-13.86 \\
-13.86\end{array}$ & $\begin{array}{l}0.034 \\
0.074\end{array}$ & $\begin{array}{l}0.959 \\
0.915\end{array}$ \\
\hline
\end{tabular}


Table 16c. Heating and Cooling Coincidence Factors for Large Hotel

\begin{tabular}{|cl|ccc|cc|}
\hline Location & Vintage & $\begin{array}{c}\Delta \mathrm{HL} \\
(\mathrm{kBtw} / \mathrm{sf})\end{array}$ & $\begin{array}{c}\Delta \mathrm{CL} \\
(\mathrm{kBtu} / \mathrm{sf})\end{array}$ & $\begin{array}{c}\Delta \mathrm{LL} \\
(\mathrm{kB} / \mathrm{k} / \mathrm{sf})\end{array}$ & HCF & CCF \\
\hline Charleston & Current & -1.03 & -7.07 & -8.97 & 0.115 & 0.788 \\
& Old & -0.99 & -7.14 & -8.97 & 0.110 & 0.796 \\
\hline Chicago & Current & -1.38 & -6.01 & -8.97 & 0.154 & 0.670 \\
" & Old & -2.13 & -5.12 & -8.97 & 0.237 & 0.571 \\
\hline Lake Charles & Current & -0.68 & -7.81 & -8.97 & 0.076 & 0.871 \\
" & Old & -0.73 & -7.73 & -8.97 & 0.081 & 0.862 \\
\hline Miami & Current & -0.04 & -8.85 & -8.97 & 0.004 & 0.987 \\
" & Old & -0.03 & -8.87 & -8.97 & 0.003 & 0.989 \\
\hline Minneapolis & Current & -1.76 & -5.31 & -8.97 & 0.196 & 0.592 \\
" & Old & -2.47 & -4.49 & -8.97 & 0.275 & 0.501 \\
\hline New York & Current & -1.25 & -6.01 & -8.97 & 0.139 & 0.670 \\
" & Old & -2.09 & -5.00 & -8.97 & 0.233 & 0.557 \\
\hline Pasadena & Current & -0.15 & -8.61 & -8.97 & 0.017 & 0.960 \\
" & Old & -0.21 & -8.53 & -8.97 & 0.023 & 0.951 \\
\hline Phoenix & Current & -0.22 & -8.58 & -8.97 & 0.025 & 0.957 \\
" & Old & -0.29 & -8.49 & -8.97 & 0.032 & 0.946 \\
\hline San Francisco & Current & -0.54 & -7.90 & -8.97 & 0.060 & 0.881 \\
" & Old & -0.83 & -7.53 & -8.97 & 0.093 & 0.839 \\
\hline Washington & Current & -0.87 & -6.77 & -8.97 & 0.097 & 0.755 \\
" & Old & -1.82 & -5.56 & -8.97 & 0.203 & 0.620 \\
\hline
\end{tabular}

Table 16d. Heating and Cooling Coincidence Factors for Large Office

\begin{tabular}{|cl|ccc|cc|}
\hline Location & Vintage & $\begin{array}{c}\Delta \mathrm{HL} \\
(\mathrm{kBtw} / \mathrm{sf})\end{array}$ & $\begin{array}{c}\Delta \mathrm{CL} \\
(\mathrm{kBtu} / \mathrm{sf})\end{array}$ & $\begin{array}{c}\Delta \mathrm{LL} \\
(\mathrm{kBtu} / \mathrm{sf})\end{array}$ & HCF & CCF \\
\hline Charleston & Current & -0.55 & -5.61 & -6.89 & 0.080 & 0.814 \\
& Old & -0.64 & -7.23 & -8.78 & 0.073 & 0.823 \\
\hline Chicago & Current & -1.54 & -4.27 & -6.58 & 0.234 & 0.649 \\
" & Old & -1.97 & -4.98 & -7.97 & 0.247 & 0.625 \\
\hline Lake Charles & Current & -0.29 & -6.02 & -6.90 & 0.042 & 0.872 \\
" & Old & -0.39 & -7.60 & -8.78 & 0.044 & 0.866 \\
\hline Miami & Current & -0.02 & -6.35 & -6.89 & 0.003 & 0.922 \\
" & Old & -0.02 & -8.07 & -8.78 & 0.002 & 0.919 \\
\hline Minneapolis & Current & -2.09 & -3.69 & -6.58 & 0.318 & 0.561 \\
" & Old & -2.66 & -4.30 & -7.97 & 0.334 & 0.540 \\
\hline New York & Current & -1.98 & -3.77 & -6.58 & 0.301 & 0.573 \\
" & Old & -2.34 & -4.17 & -7.55 & 0.310 & 0.552 \\
\hline Pasadena & Current & -0.18 & -5.46 & -6.26 & 0.029 & 0.872 \\
" & Old & -0.23 & -6.93 & -7.98 & 0.029 & 0.868 \\
\hline Phoenix & Current & -0.21 & -5.48 & -6.26 & 0.034 & 0.875 \\
" & Old & -0.26 & -6.98 & -7.98 & 0.033 & 0.875 \\
\hline San Francisco & Current & -0.58 & -4.66 & -6.26 & 0.093 & 0.744 \\
" & Old & -0.64 & -6.17 & -7.98 & 0.080 & 0.773 \\
\hline Washington & Current & -1.18 & -4.62 & -6.58 & 0.179 & 0.702 \\
" & Old & -1.48 & -5.10 & -7.55 & 0.196 & 0.675 \\
\hline
\end{tabular}


Table 16e. Heating and Cooling Coincidence Factors for Large Retail

\begin{tabular}{|c|c|c|c|c|c|c|}
\hline Location & Vintage & $\begin{array}{c}\Delta H L \\
(k B t w / s f)\end{array}$ & $\begin{array}{c}\triangle \mathrm{CL} \\
(\mathrm{kBtw} / \mathrm{st})\end{array}$ & $\begin{array}{c}\Delta \mathrm{LL} \\
(\mathrm{kBtw} / \mathrm{sf})\end{array}$ & HCF & CCF \\
\hline Charleston & $\begin{array}{l}\text { Current } \\
\text { Old }\end{array}$ & $\begin{array}{l}-1.23 \\
-1.34\end{array}$ & $\begin{array}{l}-5.55 \\
-6.52\end{array}$ & $\begin{array}{l}-7.97 \\
-9.29\end{array}$ & $\begin{array}{l}0.154 \\
0.144\end{array}$ & $\begin{array}{l}0.696 \\
0.702\end{array}$ \\
\hline Chicago & $\begin{array}{l}\text { Current } \\
\text { Old }\end{array}$ & $\begin{array}{l}-3.31 \\
-3.95\end{array}$ & $\begin{array}{l}-3.42 \\
-3.81\end{array}$ & $\begin{array}{l}-7.81 \\
-9.11\end{array}$ & $\begin{array}{l}0.424 \\
0.434\end{array}$ & $\begin{array}{l}0.438 \\
0.418\end{array}$ \\
\hline Lake Charles & $\begin{array}{l}\text { Current } \\
\text { Old }\end{array}$ & $\begin{array}{l}-0.83 \\
-0.98\end{array}$ & $\begin{array}{l}-6.02 \\
-6.92\end{array}$ & $\begin{array}{l}-7.97 \\
-9.29\end{array}$ & $\begin{array}{l}0.104 \\
0.105\end{array}$ & $\begin{array}{l}0.755 \\
0.745\end{array}$ \\
\hline Miami & $\begin{array}{l}\text { Current } \\
\text { Old }\end{array}$ & $\begin{array}{l}-0.02 \\
-0.02\end{array}$ & $\begin{array}{l}-7.08 \\
-8.20\end{array}$ & $\begin{array}{l}-7.97 \\
-9.29\end{array}$ & $\begin{array}{l}0.003 \\
0.002\end{array}$ & $\begin{array}{l}0.888 \\
0.883\end{array}$ \\
\hline Minneapolis & $\begin{array}{l}\text { Current } \\
\text { Old }\end{array}$ & $\begin{array}{l}-3.84 \\
-4.55\end{array}$ & $\begin{array}{l}-2.97 \\
-3.32\end{array}$ & $\begin{array}{l}-7.81 \\
-9.11\end{array}$ & $\begin{array}{l}0.492 \\
0.499\end{array}$ & $\begin{array}{l}0.380 \\
0.364\end{array}$ \\
\hline New York & $\begin{array}{l}\text { Current } \\
\text { Old }\end{array}$ & $\begin{array}{l}-3.58 \\
-4.15\end{array}$ & $\begin{array}{l}-4.46 \\
-5.17\end{array}$ & $\begin{array}{r}-9.05 \\
-10.56\end{array}$ & $\begin{array}{l}0.396 \\
0.393\end{array}$ & $\begin{array}{l}0.493 \\
0.490\end{array}$ \\
\hline Pasadena & $\begin{array}{l}\text { Current } \\
\text { Old }\end{array}$ & $\begin{array}{l}-0.31 \\
-0.33\end{array}$ & $\begin{array}{l}-8.52 \\
-9.96\end{array}$ & $\begin{array}{r}-9.71 \\
-11.32\end{array}$ & $\begin{array}{l}0.032 \\
0.029\end{array}$ & $\begin{array}{l}0.877 \\
0.880\end{array}$ \\
\hline Phoenix & $\begin{array}{l}\text { Current } \\
\text { Old }\end{array}$ & $\begin{array}{l}-0.37 \\
-0.44\end{array}$ & $\begin{array}{l}-8.55 \\
-9.93\end{array}$ & $\begin{array}{r}-9.70 \\
-11.32\end{array}$ & $\begin{array}{l}0.038 \\
0.039\end{array}$ & $\begin{array}{l}0.881 \\
0.877\end{array}$ \\
\hline San Francisco & $\begin{array}{l}\text { Current } \\
\text { Old }\end{array}$ & $\begin{array}{l}-1.17 \\
-2.11\end{array}$ & $\begin{array}{l}-7.27 \\
-7.27\end{array}$ & $\begin{array}{r}-9.70 \\
-11.32\end{array}$ & $\begin{array}{l}0.121 \\
0.186\end{array}$ & $\begin{array}{l}0.749 \\
0.642\end{array}$ \\
\hline Washington & $\begin{array}{l}\text { Current } \\
\text { Old }\end{array}$ & $\begin{array}{l}-3.01 \\
-3.60\end{array}$ & $\begin{array}{l}-5.00 \\
-5.65\end{array}$ & $\begin{array}{r}-9.05 \\
-10.56\end{array}$ & $\begin{array}{l}0.333 \\
0.341\end{array}$ & $\begin{array}{l}0.552 \\
0.535\end{array}$ \\
\hline
\end{tabular}

Table 16f. Heating and Cooling Coincidence Factors for Medium Offic

\begin{tabular}{|c|c|c|c|c|c|c|}
\hline Location & Vintage & $\underset{(\mathrm{kBtw} / \mathrm{sf})}{\Delta H L}$ & $\begin{array}{c}\Delta C L \\
\text { (kBtu/st) }\end{array}$ & $\underset{(\mathrm{kBtw} / \mathrm{sf})}{\Delta \mathrm{LL}}$ & HCF & CCF \\
\hline \multirow{2}{*}{ Charleston } & Current & -0.98 & -5.94 & -7.91 & 0.124 & 0.751 \\
\hline & Old & -1.19 & -8.46 & -10.98 & 0.108 & 0.770 \\
\hline \multirow{2}{*}{ Chicago } & Current & -2.55 & -4.06 & -7.55 & 0.338 & 0.538 \\
\hline & Old & -3.91 & -5.01 & -10.48 & 0.373 & 0.478 \\
\hline \multirow{2}{*}{$\begin{array}{l}\text { Lake Charles } \\
\text { " }\end{array}$} & Current & -0.64 & -6.38 & -7.91 & 0.081 & 0.807 \\
\hline & Old & -0.85 & -8.83 & -10.98 & 0.077 & 0.804 \\
\hline \multirow{2}{*}{ Miami } & Current & -0.05 & -7.14 & -7.91 & 0.006 & 0.903 \\
\hline & Old & -0.06 & -9.90 & -10.98 & 0.005 & 0.902 \\
\hline \multirow{2}{*}{ Minneapolis } & Current & -3.12 & -3.52 & -7.55 & 0.413 & 0.466 \\
\hline & Old & -4.75 & -4.32 & -10.48 & 0.453 & 0.412 \\
\hline \multirow{2}{*}{ New York } & Current & -2.68 & -3.81 & -7.55 & 0.355 & 0.505 \\
\hline & Old & -3.89 & -4.90 & -10.48 & 0.371 & 0.468 \\
\hline \multirow{2}{*}{ Pasadena } & Current & -0.41 & -5.84 & -7.19 & 0.057 & 0.812 \\
\hline & Old & -0.62 & -7.92 & -9.98 & 0.062 & 0.794 \\
\hline \multirow{2}{*}{ Phoenix } & Current & -0.36 & -6.07 & -7.19 & 0.050 & 0.844 \\
\hline & Old & -0.59 & -8.18 & -9.98 & 0.059 & 0.820 \\
\hline \multirow{2}{*}{ San Francisco } & Current & -1.14 & -4.66 & -7.19 & 0.159 & 0.648 \\
\hline & Old & -1.68 & -6.31 & -9.98 & 0.168 & 0.632 \\
\hline \multirow{2}{*}{$\begin{array}{l}\text { Washington } \\
\text { " }\end{array}$} & Current & -2.10 & -4.43 & .7 .55 & 0.278 & 0.587 \\
\hline & Old & -3.06 & -5.77 & -10.48 & 0.292 & 0.551 \\
\hline
\end{tabular}


Table 16g. Heating and Cooling Coincidence Factors for Small Hotel/Motel

\begin{tabular}{|c|c|c|c|c|c|c|}
\hline Location & Vintage & $\begin{array}{c}\Delta H L \\
(k B t w / s f)\end{array}$ & $\begin{array}{c}\Delta C L \\
(k B t u / s f)\end{array}$ & $\begin{array}{c}\Delta L L \\
(k B t w / s f)\end{array}$ & HCF & CCF \\
\hline Charleston & $\begin{array}{l}\text { Current } \\
\text { Old }\end{array}$ & $\begin{array}{l}-1.12 \\
-1.11\end{array}$ & $\begin{array}{l}-2.44 \\
-2.47\end{array}$ & $\begin{array}{l}-4.16 \\
-4.16\end{array}$ & $\begin{array}{l}0.269 \\
0.267\end{array}$ & $\begin{array}{l}0.587 \\
0.594\end{array}$ \\
\hline Chicago & $\begin{array}{l}\text { Current } \\
\text { Old }\end{array}$ & $\begin{array}{l}-1.45 \\
-1.98\end{array}$ & $\begin{array}{l}-2.26 \\
-1.75\end{array}$ & $\begin{array}{l}-4.16 \\
-4.16\end{array}$ & $\begin{array}{l}0.349 \\
0.476\end{array}$ & $\begin{array}{l}0.543 \\
0.421\end{array}$ \\
\hline $\begin{array}{l}\text { Lake Charles } \\
\text { " }\end{array}$ & $\begin{array}{l}\text { Current } \\
\text { Old }\end{array}$ & $\begin{array}{l}-0.68 \\
-0.81\end{array}$ & $\begin{array}{l}-2.92 \\
-2.74\end{array}$ & $\begin{array}{l}-4.16 \\
-4.16\end{array}$ & $\begin{array}{l}0.163 \\
0.195\end{array}$ & $\begin{array}{l}0.702 \\
0.659\end{array}$ \\
\hline Miami & $\begin{array}{l}\text { Current } \\
\text { Old }\end{array}$ & $\begin{array}{l}-0.07 \\
-0.07\end{array}$ & $\begin{array}{l}-3.87 \\
-3.86\end{array}$ & $\begin{array}{l}-4.16 \\
-4.16\end{array}$ & $\begin{array}{l}0.017 \\
0.017\end{array}$ & $\begin{array}{l}0.930 \\
0.928\end{array}$ \\
\hline Minneapolis & $\begin{array}{l}\text { Current } \\
\text { Old }\end{array}$ & $\begin{array}{l}-1.79 \\
-2.24\end{array}$ & $\begin{array}{l}-1.98 \\
-1.54\end{array}$ & $\begin{array}{l}-4.16 \\
-4.16\end{array}$ & $\begin{array}{l}0.430 \\
0.538\end{array}$ & $\begin{array}{l}0.476 \\
0.370\end{array}$ \\
\hline$\underset{n}{\text { New York }}$ & $\begin{array}{l}\text { Current } \\
\text { Old }\end{array}$ & $\begin{array}{l}-1.50 \\
-1.85\end{array}$ & $\begin{array}{l}-2.23 \\
-1.85\end{array}$ & $\begin{array}{l}-4.16 \\
-4.16\end{array}$ & $\begin{array}{l}0.361 \\
0.445\end{array}$ & $\begin{array}{l}0.536 \\
0.445\end{array}$ \\
\hline Pasadena & $\begin{array}{l}\text { Current } \\
\text { Old }\end{array}$ & $\begin{array}{l}-0.67 \\
-0.90\end{array}$ & $\begin{array}{l}-2.64 \\
-2.33\end{array}$ & $\begin{array}{l}-4.16 \\
.4 .16\end{array}$ & $\begin{array}{l}0.161 \\
0.216\end{array}$ & $\begin{array}{l}0.635 \\
0.560\end{array}$ \\
\hline Phoenix & $\begin{array}{l}\text { Current } \\
\text { Old }\end{array}$ & $\begin{array}{l}-0.48 \\
-0.63\end{array}$ & $\begin{array}{l}-3.24 \\
-3.06\end{array}$ & $\begin{array}{l}-4.16 \\
-4.16\end{array}$ & $\begin{array}{l}0.115 \\
0.151\end{array}$ & $\begin{array}{l}0.779 \\
0.736\end{array}$ \\
\hline San Francisco $_{n}$ & $\begin{array}{l}\text { Current } \\
\text { Old }\end{array}$ & $\begin{array}{l}-1.30 \\
-1.81\end{array}$ & $\begin{array}{l}-1.67 \\
-1.11\end{array}$ & $\begin{array}{l}-4.16 \\
-4.16\end{array}$ & $\begin{array}{l}0.312 \\
0.435\end{array}$ & $\begin{array}{l}0.401 \\
0.267\end{array}$ \\
\hline Washington & $\begin{array}{l}\text { Current } \\
\text { Old }\end{array}$ & $\begin{array}{l}-1.14 \\
-1.65\end{array}$ & $\begin{array}{l}-2.59 \\
-2.10\end{array}$ & $\begin{array}{l}-4.16 \\
-4.16\end{array}$ & $\begin{array}{l}0.274 \\
0.397\end{array}$ & $\begin{array}{l}0.623 \\
0.505\end{array}$ \\
\hline
\end{tabular}

Table 16h. Heating and Cooling Coincidence Factors for Sit-down Restaurant

\begin{tabular}{|ll|ccc|cc|}
\hline Location & Vintage & $\begin{array}{c}\Delta \mathrm{HL} \\
(\mathrm{kBtu} / \mathrm{sf})\end{array}$ & $\begin{array}{c}\Delta \mathrm{CL} \\
(\mathrm{kBtu} / \mathrm{sf})\end{array}$ & $\begin{array}{c}\Delta \mathrm{LL} \\
(\mathrm{kBtw} / \mathrm{s})\end{array}$ & HCF & CCF \\
\hline Charleston & Average & -3.86 & -11.64 & -16.80 & 0.230 & 0.693 \\
Chicago & Average & -7.37 & -8.49 & -16.81 & 0.438 & 0.505 \\
Lake Charles & Average & -2.89 & -12.66 & -16.80 & 0.172 & 0.754 \\
Miami & Average & -0.30 & -15.60 & -16.80 & 0.018 & 0.929 \\
Minneapolis & Average & -8.54 & -7.42 & -16.81 & 0.508 & 0.441 \\
New York & Average & -7.12 & -8.67 & -16.81 & 0.424 & 0.516 \\
Pasadena & Average & -2.95 & -12.27 & -16.82 & 0.175 & 0.729 \\
Phoenix & Average & -2.02 & -13.71 & -16.81 & 0.120 & 0.816 \\
San Francisco & Average & -5.77 & -9.09 & -16.80 & 0.343 & 0.541 \\
Washington & Average & -6.17 & -9.65 & -16.80 & 0.367 & 0.574 \\
\hline
\end{tabular}


Table 16i. Heating and Cooling Coincidence Factors for Supermarket

\begin{tabular}{|c|c|c|c|c|c|c|}
\hline Location & Vintage & $\begin{array}{c}\Delta H L \\
(k B t w / s)\end{array}$ & $\begin{array}{c}\Delta \mathrm{CL} \\
(\mathrm{kBtw} / \mathrm{sf})\end{array}$ & $\begin{array}{c}\Delta L L \\
(k B t w / s f)\end{array}$ & HCF & CCF \\
\hline Charleston & $\begin{array}{l}\text { Current } \\
\text { Old }\end{array}$ & $\begin{array}{l}-2.90 \\
-3.54 \\
\end{array}$ & $\begin{array}{l}-15.72 \\
-14.93 \\
\end{array}$ & $\begin{array}{l}-20.90 \\
-20.90 \\
\end{array}$ & $\begin{array}{l}0.139 \\
0.169 \\
\end{array}$ & $\begin{array}{l}0.752 \\
0.714 \\
\end{array}$ \\
\hline Chicago & $\begin{array}{l}\text { Current } \\
\text { Old }\end{array}$ & $\begin{array}{l}-6.23 \\
-8.76 \\
\end{array}$ & $\begin{array}{l}-12.75 \\
-10.00 \\
\end{array}$ & $\begin{array}{l}-20.90 \\
-20.90 \\
\end{array}$ & $\begin{array}{l}0.298 \\
0.419 \\
\end{array}$ & $\begin{array}{l}0.610 \\
0.478 \\
\end{array}$ \\
\hline $\begin{array}{l}\text { Lake Charles } \\
\text { " }\end{array}$ & $\begin{array}{l}\text { Current } \\
\text { Old } \\
\end{array}$ & $\begin{array}{l}-1.49 \\
-2.48 \\
\end{array}$ & $\begin{array}{l}-17.86 \\
-16.30 \\
\end{array}$ & $\begin{array}{l}-20.90 \\
-20.90 \\
\end{array}$ & $\begin{array}{l}0.071 \\
0.119 \\
\end{array}$ & $\begin{array}{l}0.855 \\
0.780 \\
\end{array}$ \\
\hline Miami & $\begin{array}{l}\text { Current } \\
\text { Old }\end{array}$ & $\begin{array}{l}-0.08 \\
-0.15\end{array}$ & $\begin{array}{l}-20.26 \\
-20.00 \\
\end{array}$ & $\begin{array}{l}-20.90 \\
-20.90\end{array}$ & $\begin{array}{l}0.004 \\
0.007\end{array}$ & $\begin{array}{l}0.969 \\
0.957\end{array}$ \\
\hline Minneapolis & $\begin{array}{l}\text { Current } \\
\text { Old }\end{array}$ & $\begin{array}{r}-7.74 \\
-10.46 \\
\end{array}$ & $\begin{array}{r}-11.21 \\
-8.54 \\
\end{array}$ & $\begin{array}{l}-20.90 \\
-20.90 \\
\end{array}$ & $\begin{array}{l}0.370 \\
0.500 \\
\end{array}$ & $\begin{array}{l}0.536 \\
0.409 \\
\end{array}$ \\
\hline New York & $\begin{array}{l}\text { Current } \\
\text { Old }\end{array}$ & $\begin{array}{l}-5.78 \\
-8.19\end{array}$ & $\begin{array}{l}-13.05 \\
-10.30\end{array}$ & $\begin{array}{l}-20.90 \\
-20.90\end{array}$ & $\begin{array}{l}0.277 \\
0.392\end{array}$ & $\begin{array}{l}0.624 \\
0.493 \\
\end{array}$ \\
\hline $\begin{array}{c}\text { Pasadena } \\
\text { " }\end{array}$ & $\begin{array}{l}\text { Current } \\
\text { Old }\end{array}$ & $\begin{array}{l}-0.88 \\
-1.74 \\
\end{array}$ & $\begin{array}{l}-17.83 \\
-16.17 \\
\end{array}$ & $\begin{array}{l}-20.90 \\
-20.90 \\
\end{array}$ & $\begin{array}{l}0.042 \\
0.083 \\
\end{array}$ & $\begin{array}{l}0.853 \\
0.774 \\
\end{array}$ \\
\hline $\begin{array}{l}\text { Phoenix } \\
\text { " }\end{array}$ & $\begin{array}{l}\text { Current } \\
\text { Old }\end{array}$ & $\begin{array}{l}-0.70 \\
-1.73 \\
\end{array}$ & $\begin{array}{l}-19.17 \\
-17.37 \\
\end{array}$ & $\begin{array}{r}-20.90 \\
-20.90 \\
\end{array}$ & $\begin{array}{l}0.033 \\
0.083 \\
\end{array}$ & $\begin{array}{l}0.917 \\
0.831 \\
\end{array}$ \\
\hline San Francisco & $\begin{array}{l}\text { Current } \\
\text { Old } \\
\end{array}$ & $\begin{array}{l}-2.03 \\
-4.63 \\
\end{array}$ & $\begin{array}{l}-15.72 \\
-11.54 \\
\end{array}$ & $\begin{array}{l}-20.90 \\
-20.90 \\
\end{array}$ & $\begin{array}{l}0.097 \\
0.222 \\
\end{array}$ & $\begin{array}{l}0.752 \\
0.552 \\
\end{array}$ \\
\hline Washington & $\begin{array}{l}\text { Current } \\
\text { Old }\end{array}$ & $\begin{array}{l}-4.66 \\
-7.16\end{array}$ & $\begin{array}{l}-14.23 \\
-11.36\end{array}$ & $\begin{array}{l}-20.90 \\
-20.90\end{array}$ & $\begin{array}{l}0.223 \\
0.343\end{array}$ & $\begin{array}{l}0.681 \\
0.544\end{array}$ \\
\hline
\end{tabular}

Table 16j. Heating and Cooling Coincidence Factors for Secondary School

\begin{tabular}{|c|c|c|c|c|c|c|}
\hline Location & Vintage & $\begin{array}{c}\Delta H L \\
(k B t w / s f)\end{array}$ & $\begin{array}{c}\Delta \mathrm{CL} \\
\text { (kBtw/sf) }\end{array}$ & $\begin{array}{c}\Delta L L \\
(k B t w / s f)\end{array}$ & HCF & CCF \\
\hline Charleston & $\begin{array}{l}\text { Current } \\
\text { Old }\end{array}$ & $\begin{array}{l}-0.86 \\
-0.94\end{array}$ & $\begin{array}{l}-3.34 \\
-3.23\end{array}$ & $\begin{array}{l}-5.15 \\
-5.15\end{array}$ & $\begin{array}{l}0.167 \\
0.183\end{array}$ & $\begin{array}{l}0.649 \\
0.627\end{array}$ \\
\hline Chicago & $\begin{array}{l}\text { Current } \\
\text { Old }\end{array}$ & $\begin{array}{l}-1.59 \\
-2.27\end{array}$ & $\begin{array}{l}-2.72 \\
-1.91\end{array}$ & $\begin{array}{l}-5.15 \\
-5.15\end{array}$ & $\begin{array}{l}0.309 \\
0.441\end{array}$ & $\begin{array}{l}0.528 \\
0.371\end{array}$ \\
\hline $\begin{array}{l}\text { Pasadena } \\
\text { " }\end{array}$ & $\begin{array}{l}\text { Current } \\
\text { Old }\end{array}$ & $\begin{array}{l}-0.39 \\
-0.54\end{array}$ & $\begin{array}{l}-3.86 \\
-3.63\end{array}$ & $\begin{array}{l}-5.15 \\
-5.15\end{array}$ & $\begin{array}{l}0.076 \\
0.105\end{array}$ & $\begin{array}{l}0.750 \\
0.705\end{array}$ \\
\hline Lake Charles & $\begin{array}{l}\text { Current } \\
\text { Old }\end{array}$ & $\begin{array}{l}-0.56 \\
-0.75\end{array}$ & $\begin{array}{l}-3.70 \\
-3.43\end{array}$ & $\begin{array}{l}-5.15 \\
-5.15\end{array}$ & $\begin{array}{l}0.109 \\
0.146\end{array}$ & $\begin{array}{l}0.718 \\
0.666\end{array}$ \\
\hline Miami & $\begin{array}{l}\text { Current } \\
\text { Old }\end{array}$ & $\begin{array}{l}-0.04 \\
-0.04\end{array}$ & $\begin{array}{l}-4.26 \\
-4.23\end{array}$ & $\begin{array}{l}-5.15 \\
-5.15\end{array}$ & $\begin{array}{l}0.008 \\
0.008\end{array}$ & $\begin{array}{l}0.827 \\
0.821\end{array}$ \\
\hline Minneapolis & $\begin{array}{l}\text { Current } \\
\text { Old }\end{array}$ & $\begin{array}{l}-2.06 \\
-2.63\end{array}$ & $\begin{array}{l}-2.28 \\
-1.55\end{array}$ & $\begin{array}{l}-5.15 \\
-5.15\end{array}$ & $\begin{array}{l}0.400 \\
0.511\end{array}$ & $\begin{array}{l}0.443 \\
0.301\end{array}$ \\
\hline New York & $\begin{array}{l}\text { Current } \\
\text { Old }\end{array}$ & $\begin{array}{l}-1.41 \\
-2.18\end{array}$ & $\begin{array}{l}-2.88 \\
-1.97\end{array}$ & $\begin{array}{l}-5.15 \\
-5.15\end{array}$ & $\begin{array}{l}0.274 \\
0.423\end{array}$ & $\begin{array}{l}0.559 \\
0.383\end{array}$ \\
\hline Phoenix & $\begin{array}{l}\text { Current } \\
\text { Old }\end{array}$ & $\begin{array}{l}-0.25 \\
-0.41\end{array}$ & $\begin{array}{l}-4.06 \\
-3.79\end{array}$ & $\begin{array}{l}-5.15 \\
-5.15\end{array}$ & $\begin{array}{l}0.049 \\
0.080\end{array}$ & $\begin{array}{l}0.788 \\
0.736\end{array}$ \\
\hline San Francisco & $\begin{array}{l}\text { Current } \\
\text { Old }\end{array}$ & $\begin{array}{l}-0.77 \\
-1.21\end{array}$ & $\begin{array}{l}-3.40 \\
-2.84\end{array}$ & $\begin{array}{l}-5.15 \\
-5.15\end{array}$ & $\begin{array}{l}0.150 \\
0.235\end{array}$ & $\begin{array}{l}0.660 \\
0.551\end{array}$ \\
\hline Washington & $\begin{array}{l}\text { Current } \\
\text { Old }\end{array}$ & $\begin{array}{l}-1.11 \\
-1.88\end{array}$ & $\begin{array}{l}-3.20 \\
-2.28\end{array}$ & $\begin{array}{l}-5.15 \\
-5.15\end{array}$ & $\begin{array}{l}0.216 \\
0.365\end{array}$ & $\begin{array}{l}0.621 \\
0.443\end{array}$ \\
\hline
\end{tabular}


Figure 1. Heating and Cooling Colncidence Factors for Lighting

Fast Food Restaurant
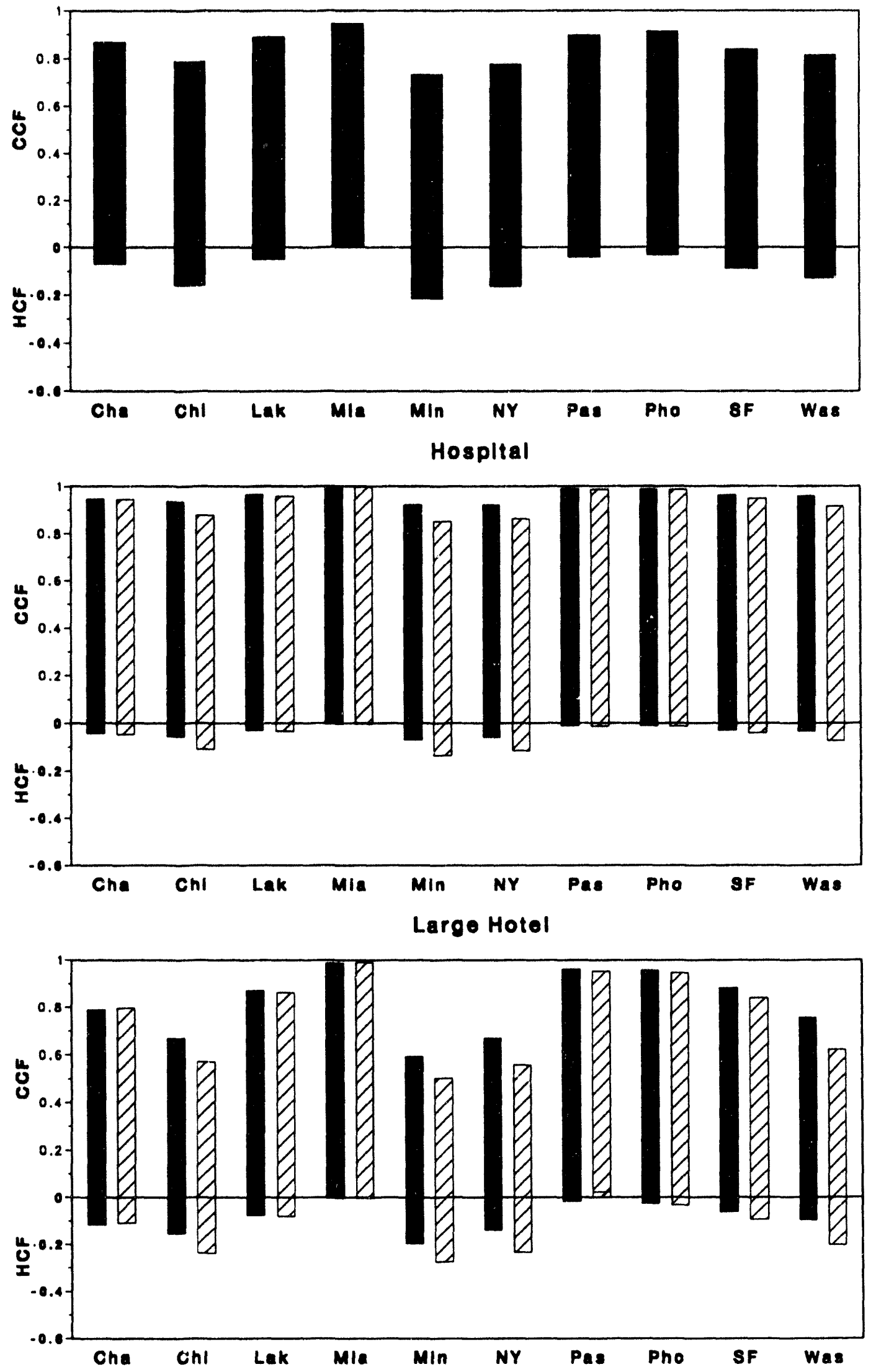

Legend

Old Vintage

C Current vintage

Cha=charleston

Chl=Chleago

Lak =Lake Charles

Mla $=$ Miami

Min=Minneapolls

NY=New York

Pas =Pasedene

Phx =Phoenlx

SFisen Francleco

Was =Washington 


$$
\frac{\|\|_{\|\|\|\|}}{\|\|\left\|_{\|\|}\right\| \|}
$$


Figure 1. Heating and Coollng Colncldence Factors for Lighting (cont.)

Small Hotel/Motel

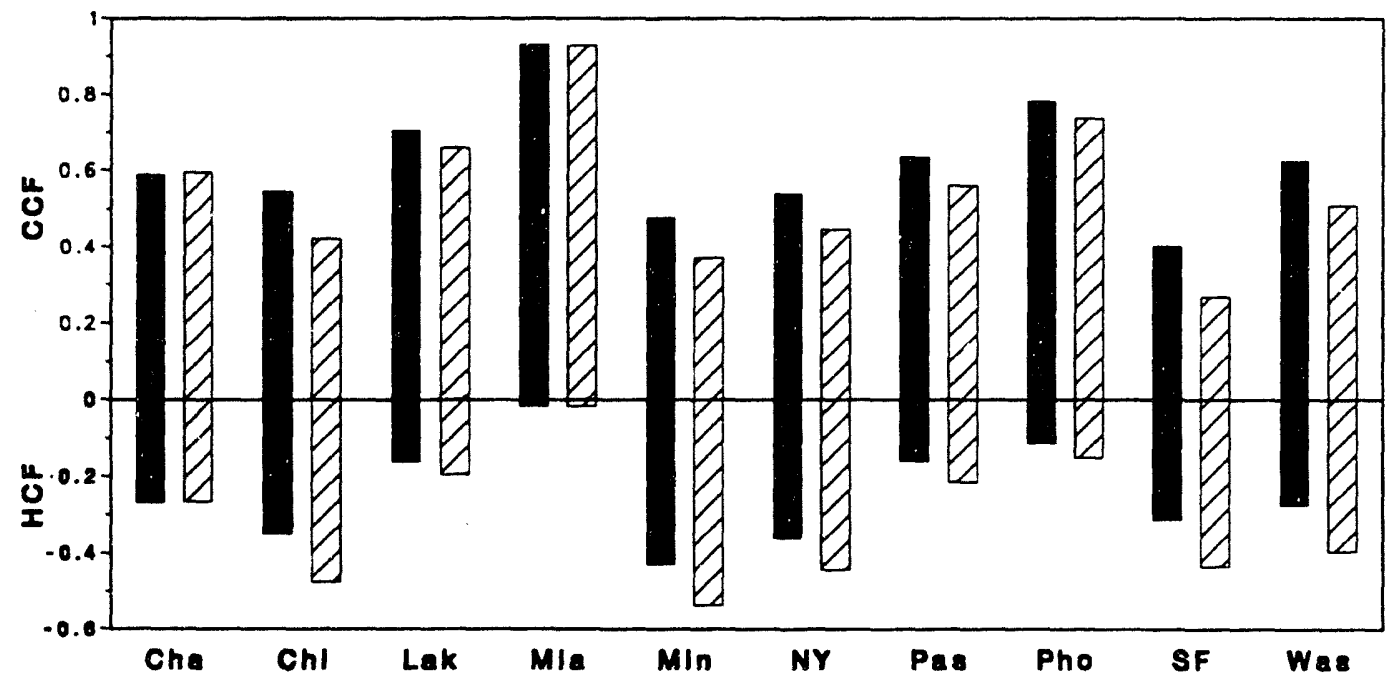

Legend

old Vintage

[ Current Vintage

Sit-down Restaurant

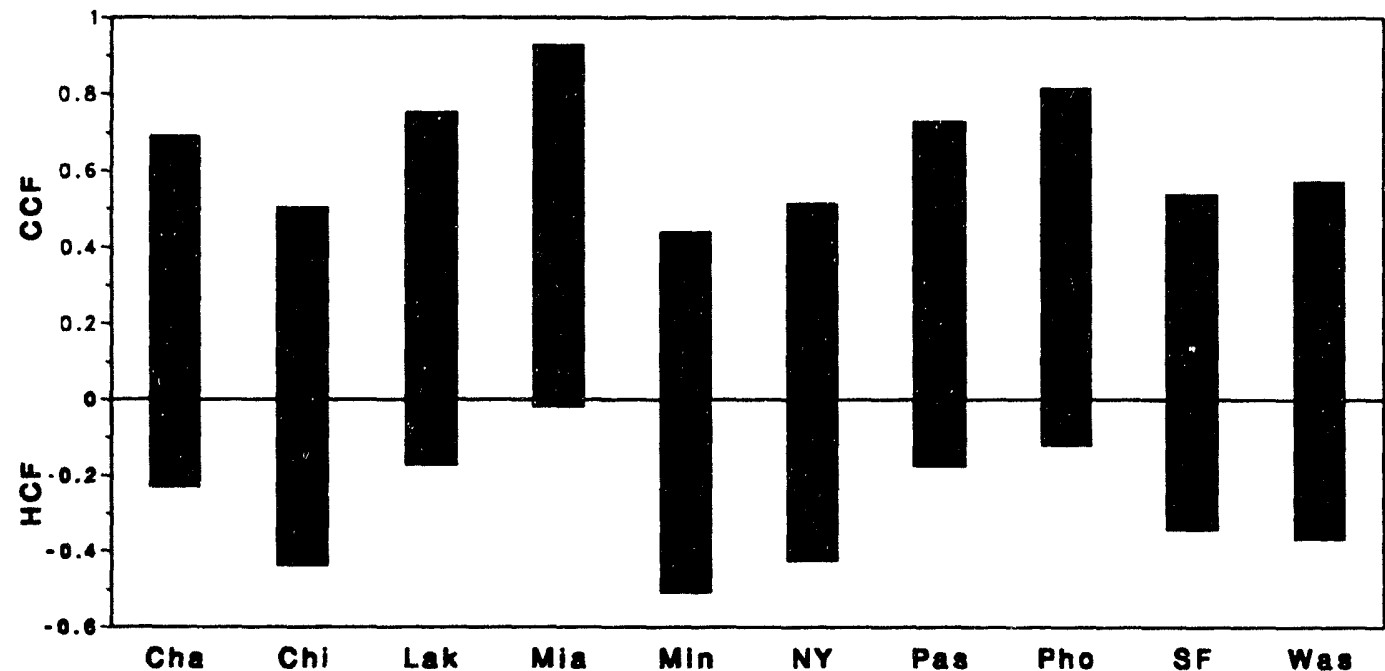

Legend

avorago Vintago

Supermarket $18 \mathrm{hr}$

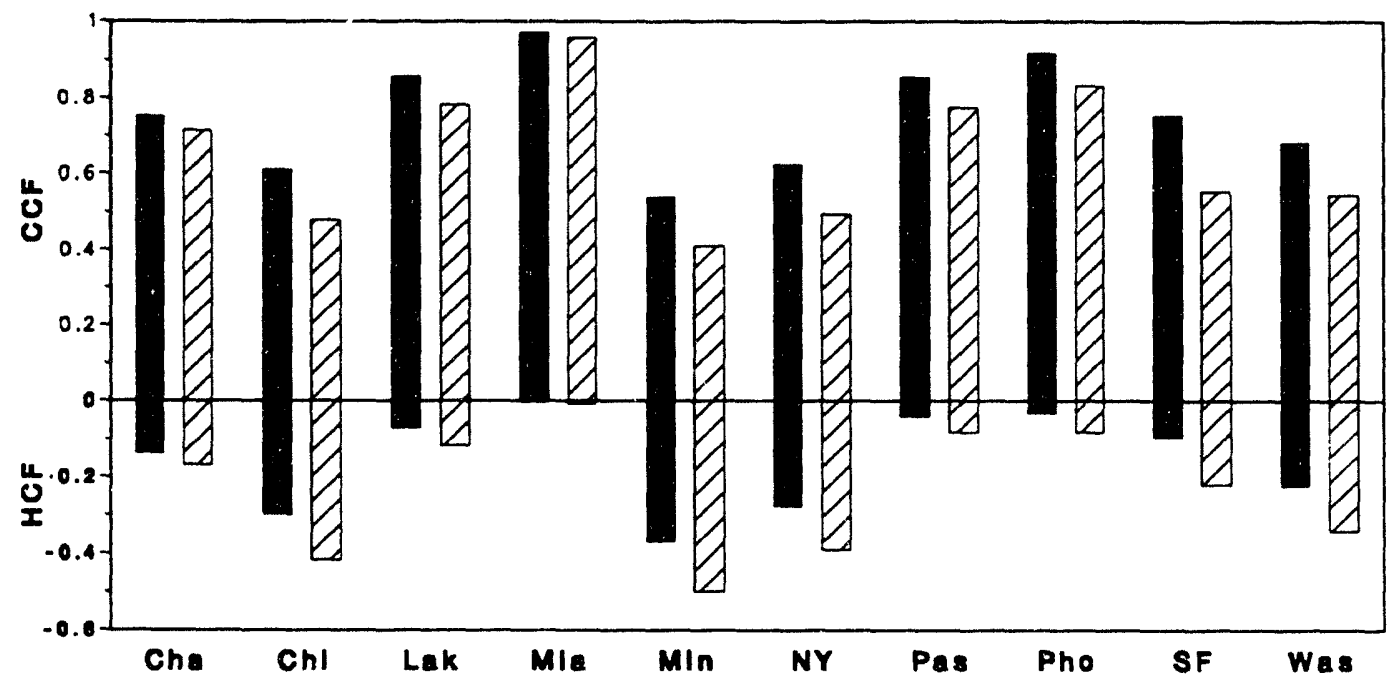

Legend

Old Vintago

Current Vintage

Cha = Charleston

Chl=Chlcago

Lak=Lake Charles

Mla = Mlam!

MIn=MInneapolls

$N Y=N$ ow York

Pas =Pasadens

Phx =Phoe $n$ lx

$S F=S a n$ Franclsco

Was=Washington 
Figure 1. Heating and Cooling Coincidence Factors for Lighting (cont.)

Secondary School

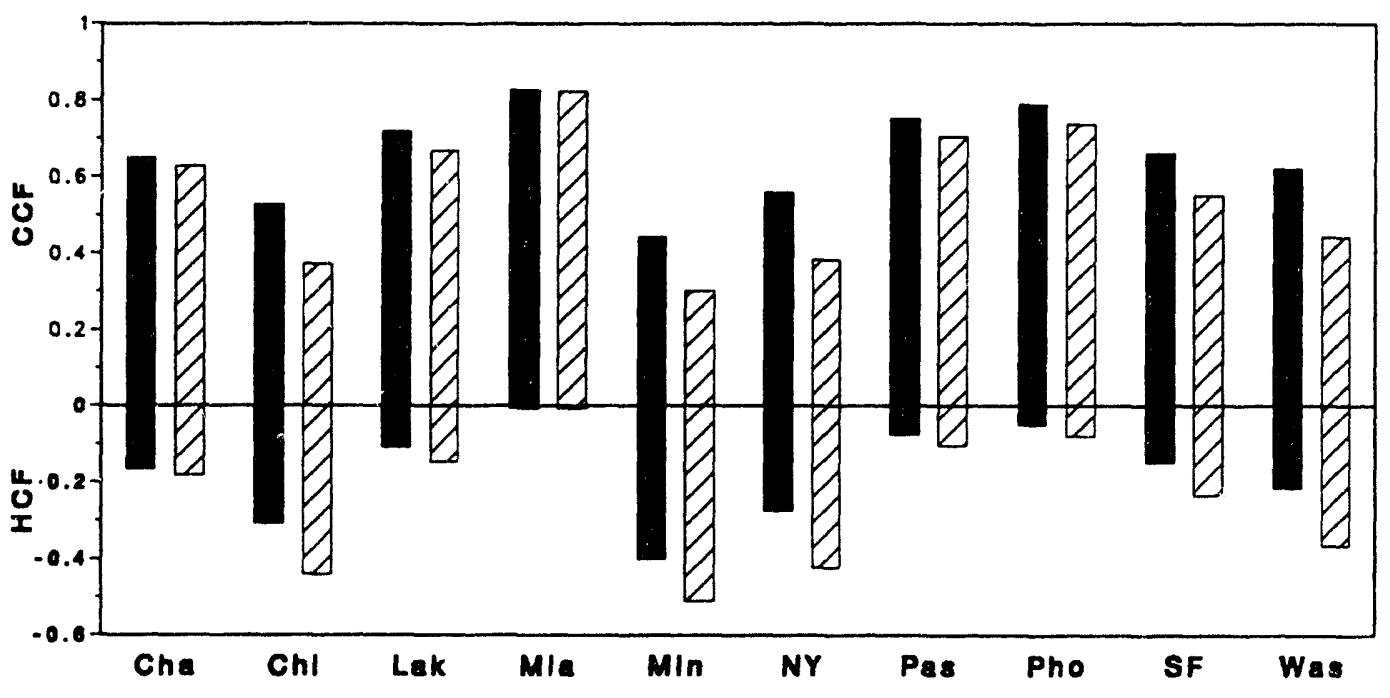

Legend old vintage $\varnothing$ Current vintage

Cha = Charleston

$\mathrm{Chl}=\mathrm{Chlcago}$

Lak=Lake Charles

MIa = Miami

MIn=MInnoepolls
NY=Now York

Pas =Pasedone

Phx=Phoonlx

SF=San Francisco
Was= Washington

50 


\section{CONCLUSIONS}

In this study, data characterizing lighting technologies for commercial buildings were developed. Together with data from parallel projects developing data for space conditioning [1], these data facilitate more detailed national level policy analysis using COMMEND 4.0.

Detailed technology representation is currently available only for space-conditioning and lighting end uses in COMMEND 4.0. Extension of such representation to refrigeration and office-equipment end uses is underway by RER. 


\section{REFERENCES}

(1) Sezgen, A.O., E.M. Franconi, S.E. Greenberg, J.G. Koomey, and H. Akbari. 1994. Technology Data Characterizing Space Conditioning in Office Buildings: Application to End-Use Forecasting with COMMEND 4.0. LBL-34123, Lawrence Berkeley Laboratory, Berkeley, California. May.

(2) Electric Power Research Institute. 1993. COMMEND 4.0 User's Guide. Electric Power Research Institute, Palo Alto, California.

(3) B.A. Atkinson, J.E. McMahon, E. Mills, P. Chan, T.W. Chan, J.H. Eto, J.D. Jennings, J.G. Koomey, K.W. Lo, M. Lecar, L. Price, F. Rubinstein, O. Sezgen, and T. Wenzel. 1992. Analysis of Federal Policy Options for Improving U.S. Lighting Energy Efficiency: Commercial and Residential Buildings. LBL-31469, Lawrence Berkeley Laboratory, Berkeley, California.

(4) U.S. Department of Energy/ Energy Information Administration. 1992. Commercial Buildings Energy Consumption Survey: Characteristics of Commercial Buildings 1989. DOE/EIA-0246(89), U.S. Department of Energy, Washington, D.C.

(5) U.S. Department of Energy/ Energy Information Administration. 1988. Nonresidential Buildings Energy Consumption Survey: Characteristics of Commercial Buildings 1986. DOE/EIA-0246(86), U.S. Department of Energy, Washington, D.C.

(6) U.S. Department of Energy/ Energy Information Administration. 1992. Lighting in Commercial Buildings. DOE/EIA-0555(92)/1, U.S. Department of Energy, Washington, D.C.

(7) Illuminating Engineering Society of North America. 1987. IES Lighting Handbook 1987: Applications Volume. Illuminating Engineering Society of North America, New York, N.Y.

(8) H. Akbari, I. Turiel, J. Eto, K. Heinemeier, B. Lebot, and L. Rainer. 1990. "A Review of Existing Commercial Energy Use Intensity and Load-Shape Studies." ACEEE 1990 Summer Study on Energy Efficiency in Buildings, vol. 3, pp3.7-3.18. American Council for an Energy Efficient Economy, Washington, D.C.

(9) ADM Associates, Inc. 1986. CEC Commercial On-Site Survey Database, PG\&E Selected Sample of Commercial Premises. ADM Associates, Sacramento, California.

(10) Lighting Research Institute and Plexus Research. 1991. Survey and Forecast of Marketplace Supply and Demand for Energy-Efficient Lighting Products. Phase I Report, Electric Power Reseach Institute, Palo Alto, California.

(The report contains projected supply and demand of lighting products from surveys of lamp and ballast manufacturers, utility DSM program planners, and lighting maintenance companies.) 
(11) U.S. Department of Commerce, Bureau of Census, 1982 to 1989. Current Industrial Report Series for Electric Lamps, Fluorescent Lamp Ballasts and Fixtures. U.S. Department of Commerce, Bureau of Census, Industry Division, Washington, D.C.

(12) Geller, H.S. and P.M. Miller. 1988. 1988 Lighting Ballast Efficiency Standards: Analysis of Electric and Economic Savings. American Council for an EnergyEfficient Economy, Washington, D.C.

(13) Huang,Y.J., H. Akbari, L. Rainer, and R. Ritschard. 1990. 481 Prototypical Commercial Buildings for Twenty Urban Market Areas. (Technical documentation of building loads data base developed for the GRI Cogeneration Market Assessment Model), LBL-29798, Lawrence Berkeley Laboratory, Berkeley, California. 

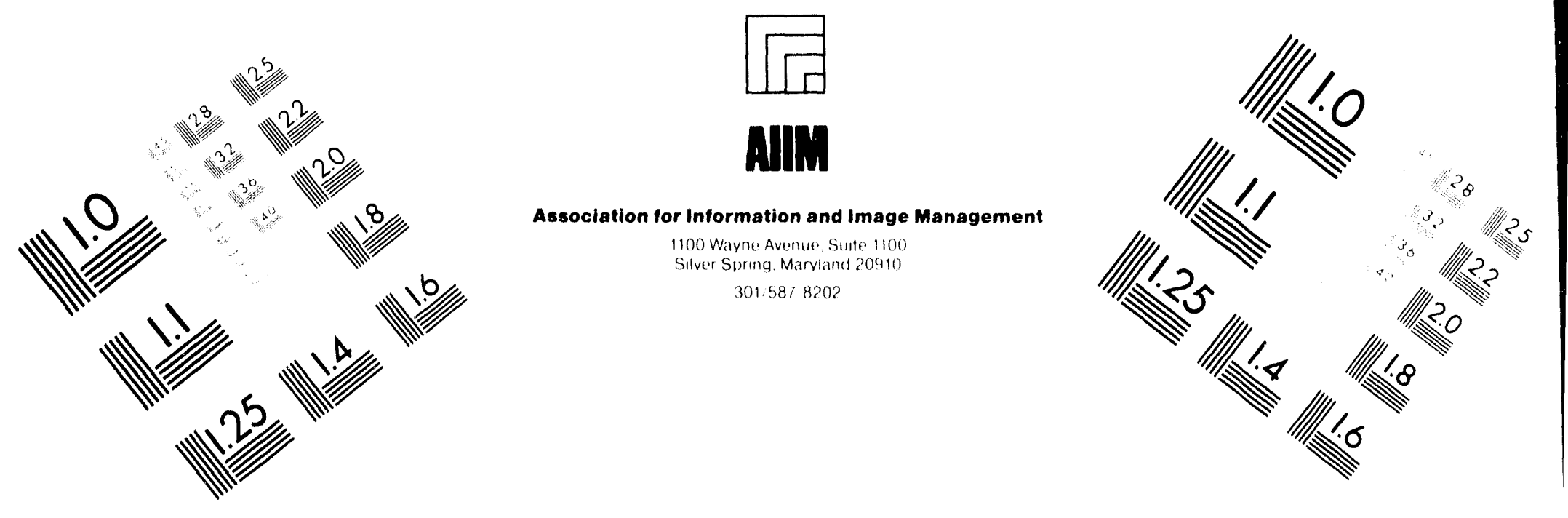

\section{Centimeter}

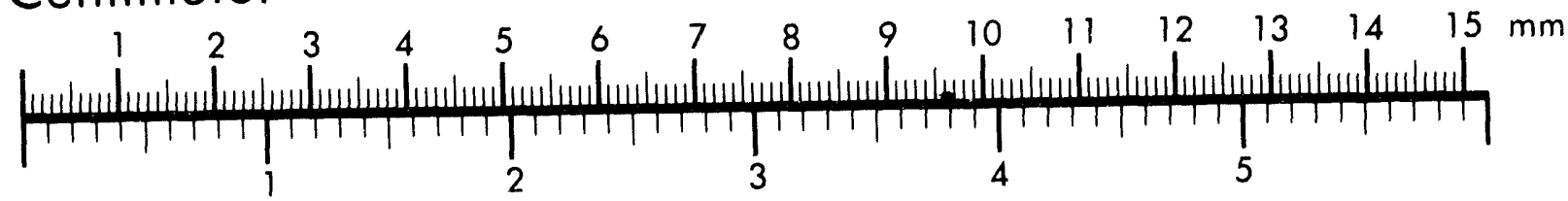
Inches
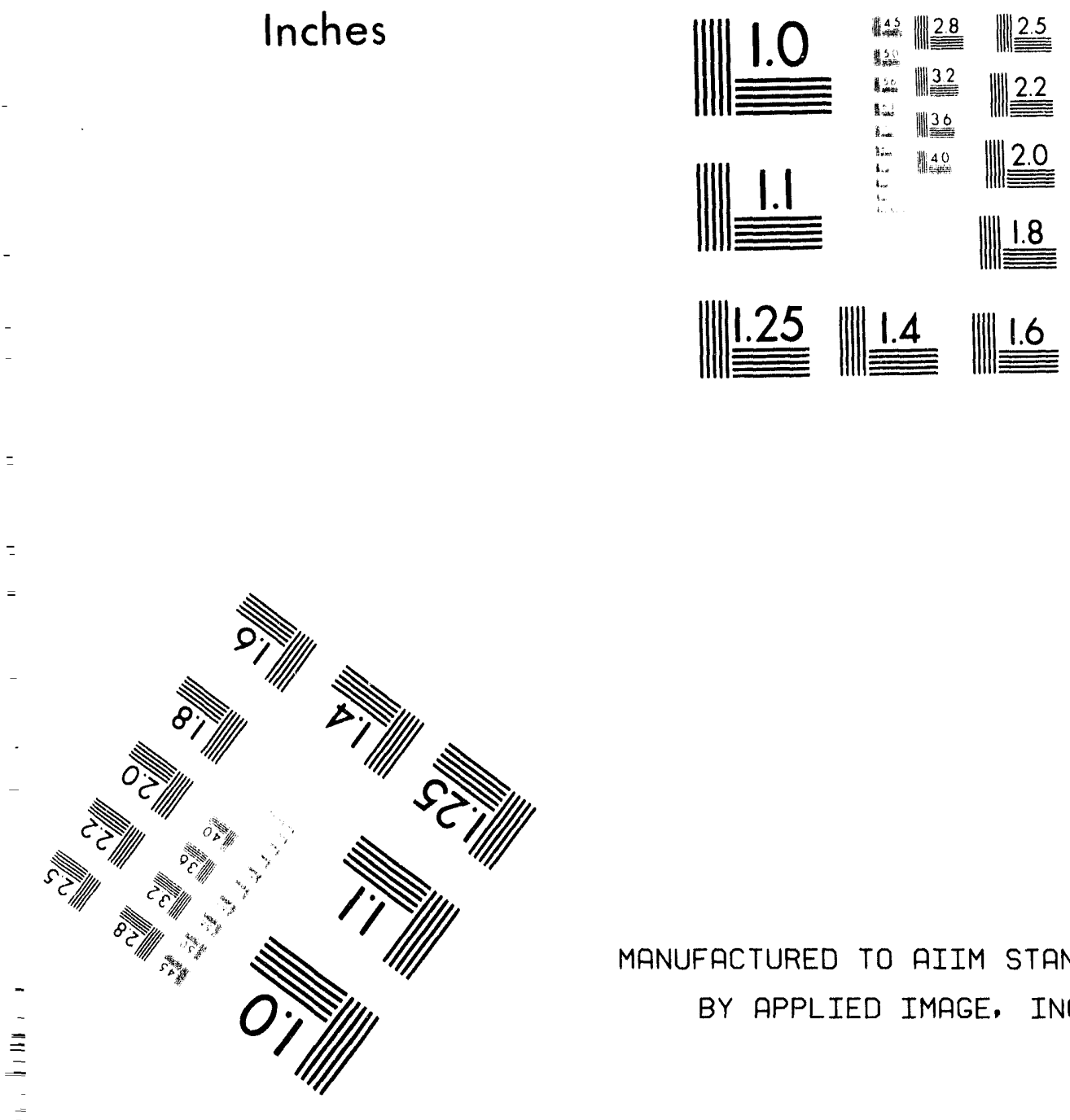

MANUFACTURED TO AIIM STANDARDS BY APPLIED IMAGE, INC.

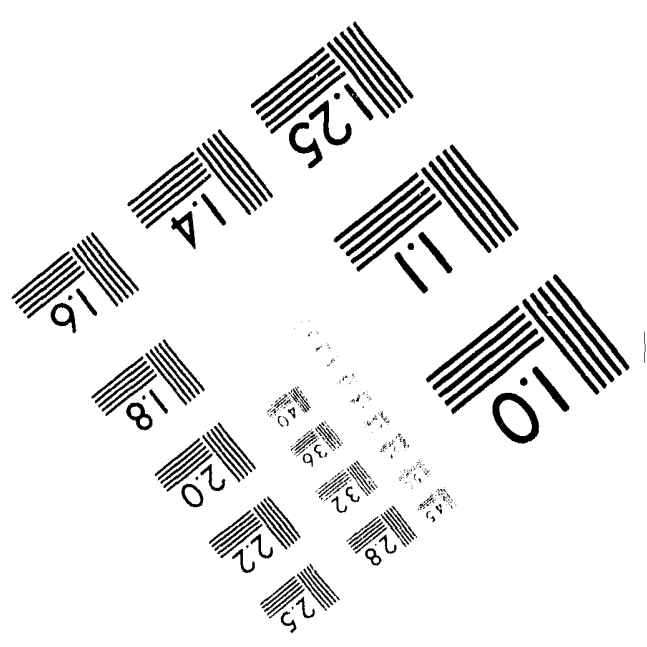



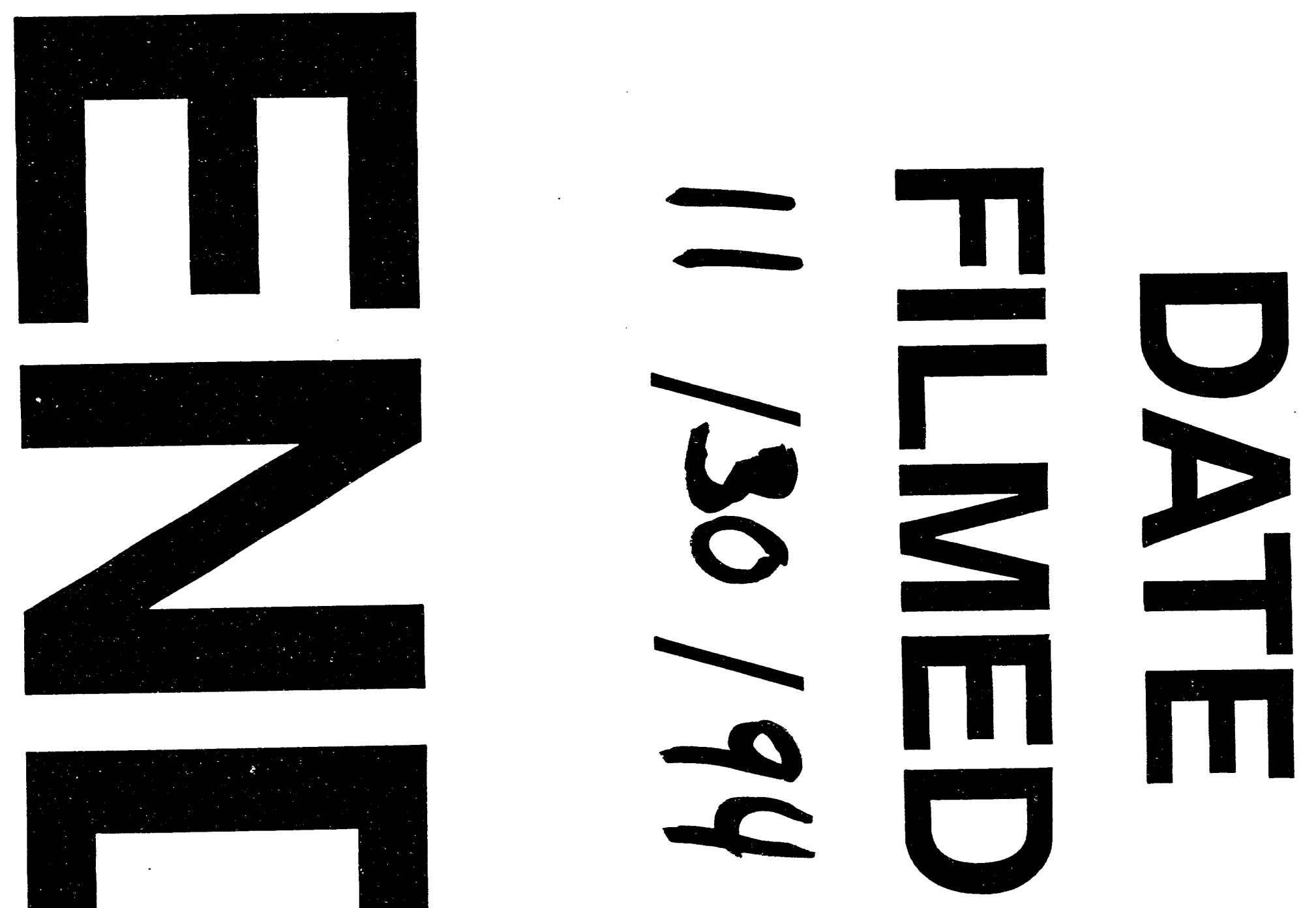